\title{
Dualizing complexes and perverse modules over differential algebras
}

\author{
Amnon Yekutieli and James J. Zhang
}

\begin{abstract}
A differential algebra of finite type over a field $\mathbb{k}$ is a filtered algebra $A$, such that the associated graded algebra is finite over its center, and the center is a finitely generated $\mathbb{k}$-algebra. The prototypical example is the algebra of differential operators on a smooth affine variety, when char $\mathbb{k}=0$. We study homological and geometric properties of differential algebras of finite type. The main results concern the rigid dualizing complex over such an algebra $A$ : its existence, structure and variance properties. We also define and study perverse $A$-modules, and show how they are related to the Auslander property of the rigid dualizing complex of $A$.
\end{abstract}

\section{Introduction}

The 'classical' Grothendieck Duality theory, dealing with dualizing complexes over schemes, was developed in the book Residues and Duality by Hartshorne [Ha66]. A duality theory for noncommutative noetherian algebras over a base field $\mathbb{k}$ was introduced in [Ye92]. Roughly speaking, a dualizing complex over a $\mathbb{k}$-algebra $A$ is a complex $R \in \mathrm{D}^{\mathrm{b}}\left(\operatorname{Mod} A^{\mathrm{e}}\right)$, such the functor

$$
\mathrm{RHom}_{A}(-, R): \mathrm{D}_{\mathrm{f}}^{\mathrm{b}}(\operatorname{Mod} A) \rightarrow \mathrm{D}_{\mathrm{f}}^{\mathrm{b}}\left(\operatorname{Mod} A^{\mathrm{op}}\right)
$$

is a duality, with inverse $\operatorname{RHom}_{A^{\mathrm{op}}}(-, R)$. Here $A^{\mathrm{op}}$ is the opposite algebra, $A^{\mathrm{e}}:=A \otimes_{\mathbb{k}} A^{\mathrm{op}}$, and $\mathrm{D}_{\mathrm{f}}^{\mathrm{b}}(\operatorname{Mod} A)$ is the derived category of bounded complexes of $A$-modules with finite cohomologies. See Definition 4.1 for details. In the decade since its introduction, this noncommutative duality theory has progressed in several directions; cf. [vdB97, Jo98, MY01, WZ00, Ch04].

One of the biggest problems in noncommutative duality theory is existence of dualizing complexes. The most effective existence criterion to date is due to Van den Bergh [vdB97]. It states the following: suppose the $\mathbb{k}$-algebra $A$ has some exhaustive nonnegative filtration $G=\left\{G_{i} A\right\}$ such that the graded algebra $\operatorname{gr}^{G} A$ is connected, noetherian and commutative. Then $A$ has a rigid dualizing complex $R_{A}$ (see Definition 4.10). Moreover, in [YZ99] we proved that the dualizing complex $R_{A}$ has the Auslander property (see Definition 4.6), and it is unique up to a unique isomorphism.

Let us remind the reader that a graded $\mathbb{k}$-algebra $\bar{A}$ is called connected if $\bar{A}=\bigoplus_{i \in \mathbb{N}} \bar{A}_{i}, \bar{A}_{0}=\mathbb{k}$ and each $\bar{A}_{i}$ is a finite $\mathbb{k}$-module. An exhaustive nonnegative filtration $G$ on $A$, such that the graded algebra $\bar{A}:=\operatorname{gr}^{G} A$ is a noetherian connected graded $\mathbb{k}$-algebra, can be considered as a 'noncommutative compactification of Spec $A$ '. Indeed, when $A$ is commutative, let $\widetilde{A}:=\operatorname{Rees}^{G} A \subset$ $A[t]$ be the Rees algebra. Then Proj $\widetilde{A}$ is a projective scheme over $\mathbb{k}$, the divisor $\{t=0\}$ is ample, and its complement is isomorphic to $\operatorname{Spec} A$.

Received 20 December 2003, accepted in final form 2 July 2004, published online 21 April 2005.

2000 Mathematics Subject Classification 16D90 (primary), 18G10, 16S32, 16W70, 16 U20 (secondary).

Keywords: noncommutative rings, filtered rings, dualizing complexes.

This research was supported by the US-Israel Binational Science Foundation. The second author was partially supported by the US National Science Foundation.

This journal is (c) Foundation Compositio Mathematica 2005. 


\section{DifFERENTIAL ALGEBRAS}

We have observed that often in the literature one encounters algebras $A$ that are equipped with an exhaustive nonnegative filtration $F=\left\{F_{i} A\right\}$, such that $\operatorname{gr}^{F} A$ is noetherian and finite over its center, yet is not connected. The main goal of this paper is to prove that Van den Bergh's existence criterion also applies to such algebras, and furthermore the rigid dualizing complex $R_{A}$ has especially good homological and geometric properties. These properties shall be used in our sequel paper [YZ02] to construct rigid dualizing complexes over noncommutative ringed schemes.

Let us now introduce some conventions. Throughout the paper $\mathbb{k}$ is a field. By default all $\mathbb{k}$-algebras are associative unital algebras, all modules are left modules, and all bimodules are central over $\mathbb{k}$. Given a $\mathbb{k}$-algebra $A$, we denote by $\operatorname{Mod} A$ the category of $A$-modules. The unadorned tensor product $\otimes$ will mean $\otimes_{\mathbb{k}}$.

Let $C$ be a finitely generated commutative $\mathbb{k}$-algebra and let $A$ be a $C$-ring (i.e. there is a ring homomorphism $C \rightarrow A$ ). We call $A$ a differential $C$-ring of finite type if there exists a nonnegative exhaustive filtration $F=\left\{F_{i} A\right\}_{i \in \mathbb{Z}}$ of $A$ such that $\operatorname{gr}^{F} A$ is a finite module over its center $\mathrm{Z}\left(\operatorname{gr}^{F} A\right)$, and $\mathrm{Z}\left(\operatorname{gr}^{F} A\right)$ is a finitely generated $C$-algebra. We also call $A$ a differential $\mathbb{k}$-algebra of finite type. See Definitions 2.1 and 2.2 for a precise formulation. The prototypical examples are:

(1) $A$ is a finite $C$-algebra (e.g. an Azumaya algebra);

(2) $A$ is the $\operatorname{ring} \mathcal{D}(C)$ of differential operators of $C$, where $C$ is smooth and char $\mathbb{k}=0$; and

(3) $A$ is the universal enveloping algebra $\mathrm{U}(C ; L)$ of a finite Lie algebroid $L$ over $C$.

In (1) and (3) there are no regularity assumptions on $C, A$ or $L$. It is not hard to see that any quotient $A / I$ is also a differential $\mathbb{k}$-algebra of finite type. Also if $A_{1}$ and $A_{2}$ are differential $\mathbb{k}$-algebras of finite type then so is the tensor product $A_{1} \otimes A_{2}$.

The key technical result is the following 'Theorem on the Two Filtrations'.

Theorem 0.1. Let $A$ be a differential $\mathbb{k}$-algebra of finite type. Then there is a nonnegative exhaustive filtration $G=\left\{G_{i} A\right\}_{i \in \mathbb{Z}}$ of $A$ such that $\mathrm{gr}^{G} A$ is a commutative, finitely generated, connected graded $\mathbb{k}$-algebra.

Theorem 0.1 is proved in $\S 3$, where it is restated as Theorem 3.1.

Since Van den Bergh's criterion can now be applied, and using results from [YZ99], we obtain the following corollary.

Corollary 0.2. Let $A$ be a differential $\mathbb{k}$-algebra of finite type. Then $A$ has an Auslander rigid dualizing complex $R_{A}$. For any finite $A$-module $M$ one has $\operatorname{Cdim} M=\operatorname{GKdim} M$.

The corollary is proved in $\S 8$, where it is restated as Theorem 8.1. We remind that GKdim $M$ is the Gelfand-Kirillov dimension of $M$. The canonical dimension Cdim $M$ is defined by

$$
\operatorname{Cdim} M:=-\inf \left\{q \mid \operatorname{Ext}_{A}^{q}\left(M, R_{A}\right) \neq 0\right\} \in \mathbb{Z} \cup\{-\infty\}
$$

for a finite $A$-module $M$, and by

$$
\mathrm{Cdim} M:=\sup \left\{\mathrm{Cdim} M^{\prime} \mid M^{\prime} \subset M \text { is finite }\right\}
$$

in general. The Auslander property states that $\operatorname{Cdim} M^{\prime} \leqslant \operatorname{Cdim} M$ for any finite left or right $A$-module $M$ and any submodule $M^{\prime} \subset M$; and it implies that Cdim is an exact dimension function.

The rigid perverse t-structure on $\mathrm{D}_{\mathrm{f}}^{\mathrm{b}}(\operatorname{Mod} A)$ is defined as follows:

$$
{ }^{p} \mathrm{D}_{\mathrm{f}}^{\mathrm{b}}(\operatorname{Mod} A)^{\leqslant 0}:=\left\{M \in \mathrm{D}_{\mathrm{f}}^{\mathrm{b}}(\operatorname{Mod} A) \mid \mathrm{H}^{i} \operatorname{RHom}_{A}\left(M, R_{A}\right)=0 \text { for all } i<0\right\}
$$

and

$$
{ }^{p} \mathrm{D}_{\mathrm{f}}^{\mathrm{b}}(\operatorname{Mod} A)^{\geqslant 0}:=\left\{M \in \mathrm{D}_{\mathrm{f}}^{\mathrm{b}}(\operatorname{Mod} A) \mid \mathrm{H}^{i} \operatorname{RHom}_{A}\left(M, R_{A}\right)=0 \text { for all } i>0\right\} .
$$




\section{A. YeKutieli And J. J. Zhang}

The heart

$$
{ }^{p} \mathrm{D}_{\mathrm{f}}^{\mathrm{b}}(\operatorname{Mod} A)^{0}:={ }^{p} \mathrm{D}_{\mathrm{f}}^{\mathrm{b}}(\operatorname{Mod} A)^{\leqslant 0} \cap{ }^{p} \mathrm{D}_{\mathrm{f}}^{\mathrm{b}}(\operatorname{Mod} A) \geqslant 0
$$

is called the category of perverse $A$-modules. It is an abelian category, dual to the category $\operatorname{Mod}_{\mathrm{f}} A^{\mathrm{op}}$ of finite $A^{\mathrm{op}}$-modules.

There is an alternative characterization of the rigid perverse t-structure on $\mathrm{D}_{\mathrm{f}}^{\mathrm{b}}(\operatorname{Mod} A)$, which resembles the original definition in [BBD81]. For a module $M$ and any integer $i$, define $\Gamma_{\mathrm{M}_{i}} M$ to be the largest submodule of $M$ with $\operatorname{Cdim} \leqslant i$. This is a functor $\Gamma_{\mathrm{M}_{i}}: \operatorname{Mod} A \rightarrow \operatorname{Mod} A$, and we denote by $\mathrm{H}_{\mathrm{M}_{i}}^{j}$ its $j$ th right derived functor. The next result is a special case of Theorem 7.9.

Theorem 0.3. Let $A$ be a differential $\mathbb{k}$-algebra of finite type and $M \in \mathrm{D}_{\mathrm{f}}^{\mathrm{b}}(\operatorname{Mod} A)$.

(1) $M \in{ }^{p} \mathrm{D}_{\mathrm{f}}^{\mathrm{b}}(\operatorname{Mod} A)^{\leqslant 0}$ if and only if $\mathrm{Cdim}^{j} M<i$ for all integers $i, j$ such that $j>-i$.

(2) $M \in{ }^{p} \mathrm{D}_{\mathrm{f}}^{\mathrm{b}}(\operatorname{Mod} A)^{\geqslant 0}$ if and only if $\mathrm{H}_{\mathrm{M}_{i}}^{j} M=0$ for all integers $i, j$ such that $j<-i$.

Interestingly, Kashiwara [Ka03] has recently proved a similar result; see Remark 7.11.

Suppose $C$ is a finitely generated commutative $\mathbb{k}$-algebra. A $C$-bimodule $M$ is called a differential $C$-bimodule if it has some bounded below exhaustive filtration $F=\left\{F_{i} M\right\}_{i \in \mathbb{Z}}$ by $C$-sub-bimodules, such that $\operatorname{gr}^{F} M$ is a central $C$-bimodule. This equivalent to the condition that the support of the $C^{\mathrm{e}}$-module $M$ is in the diagonal $\Delta(U) \subset U^{2}$, where $U:=\operatorname{Spec} C$ (see Proposition 5.21).

Theorem 0.4. Let $C$ be a finitely generated commutative $\mathbb{k}$-algebra and $A$ a differential $C$-ring of finite type. Let $R_{A}$ be the rigid dualizing complex of $A$. Then for every $i$ the cohomology bimodule $\mathrm{H}^{i} R_{A}$ is a differential C-bimodule.

This theorem is repeated as Theorem 8.14. One consequence is that the rigid dualizing complex $R_{A}$ localizes on $\operatorname{Spec} C$ (see Corollary 8.15).

Let $A^{\mathrm{e}}:=A \otimes A^{\mathrm{op}}$. It is also a differential $\mathbb{k}$-algebra of finite type, so it has a rigid dualizing complex $R_{A^{\mathrm{e}}}$, and the category ${ }^{p} \mathrm{D}_{\mathrm{f}}^{\mathrm{b}}\left(\operatorname{Mod} A^{\mathrm{e}}\right)^{0}$ of perverse $A^{\mathrm{e}}$-modules exists. By definition the rigid dualizing complex $R_{A}$ of $A$ is an object of $\mathrm{D}^{\mathrm{b}}\left(\operatorname{Mod} A^{\mathrm{e}}\right)$.

Theorem 0.5. Let $A$ be a differential $\mathbb{k}$-algebra of finite type. Then the rigid dualizing complex $R_{A}$ is a perverse $A^{\mathrm{e}}$-module, i.e. $R_{A} \in{ }^{p} \mathrm{D}_{\mathrm{f}}^{\mathrm{b}}\left(\operatorname{Mod} A^{\mathrm{e}}\right)^{0}$.

This theorem is repeated as Theorem 8.9. In addition to being interesting in itself, this result is used in [YZ02] to glue rigid dualizing complexes on noncommutative ringed schemes - as perverse bimodules.

\section{Filtrations of rings}

By a filtration of a $\mathbb{k}$-algebra $A$ we mean an ascending filtration $F=\left\{F_{i} A\right\}_{i \in \mathbb{Z}}$ by $\mathbb{k}$-submodules such that $1 \in F_{0} A$ and $F_{i} A \cdot F_{j} A \subset F_{i+j} A$. We call $(A, F)$ a filtered $\mathbb{k}$-algebra, but often we just say that $A$ is a filtered algebra and leave $F$ implicit.

Suppose $(A, F)$ is a filtered $\mathbb{k}$-algebra. Given an $A$-module $M$, by an $(A, F)$-filtration of $M$ we mean an ascending filtration $F=\left\{F_{i} M\right\}_{i \in \mathbb{Z}}$ of $M$ by $\mathbb{k}$-submodules such that $F_{i} A \cdot F_{j} M \subset F_{i+j} M$ for all $i$ and $j$. We call $(M, F)$ a filtered $(A, F)$-module, and allow ourselves to drop reference to $F$ when no confusion may arise.

We say that the filtration $F$ on $M$ is exhaustive if $M=\bigcup_{i} F_{i} M, F$ is separated if $0=\bigcap_{i} F_{i} M$, $F$ is bounded below if $F_{i_{0}-1} M=0$ for some integer $i_{0}$, and $F$ is nonnegative if $F_{-1} M=0$. The trivial filtration on $M$ is $F_{-1} M:=0, F_{0} M:=M$. 


\section{DifFERENTIAL ALGEBRAS}

Let us recall some facts about associated graded modules, and establish some notation. It will be convenient to use the ordered semigroup $\mathbb{Z} \cup\{-\infty\}$ where $-\infty<i$ for every $i \in \mathbb{Z}$, and $i+j:=-\infty$ if either $i=-\infty$ or $j=-\infty$.

Let $(M, F)$ be an exhaustive filtered module. The associated graded module is

$$
\operatorname{gr}(M, F)=\operatorname{gr}^{F} M=\bigoplus_{i \in \mathbb{Z}} \operatorname{gr}_{i}^{F} M:=\bigoplus_{i \in \mathbb{Z}} \frac{F_{i} M}{F_{i-1} M} .
$$

Given an element $m \in M$ the $F$-degree of $m$ is

$$
\operatorname{deg}^{F}(m):=\inf \left\{i \mid m \in F_{i} M\right\} \in \mathbb{Z} \cup\{-\infty\} .
$$

The $F$-symbol of $m$ is

$$
\operatorname{symb}^{F}(m):=m+F_{i-1} M \in \operatorname{gr}_{i}^{F} M
$$

if $i=\operatorname{deg}^{F}(m) \in \mathbb{Z}$; and $\operatorname{symb}^{F}(m):=0$ if $\operatorname{deg}^{F}(m)=-\infty$. Thus, the homogeneous elements of $\operatorname{gr}^{F} M$ are the symbols.

Recall that the product on the graded algebra $\operatorname{gr}^{F} A$ is defined on symbols as follows. Given elements $a_{1}, a_{2} \in A$ let $d_{i}:=\operatorname{deg}^{F}\left(a_{i}\right)$ and $\bar{a}_{i}:=\operatorname{symb}^{F}\left(a_{i}\right)$. If both $d_{i}>-\infty$ then

$$
\bar{a}_{1} \cdot \bar{a}_{2}:=a_{1} \cdot a_{2}+F_{d_{1}+d_{2}-1} A \in \operatorname{gr}_{d_{1}+d_{2}}^{F} A .
$$

Otherwise $\bar{a}_{1} \cdot \bar{a}_{2}:=0$. Similarly one defines a graded $\left(\operatorname{gr}^{F} A\right)$-module structure on a filtered module $M$.

If $A=\bigoplus_{i \in \mathbb{Z}} A_{i}$ is a graded algebra then $A$ is also filtered, where

$$
F_{i} A:=\bigoplus_{j \leqslant i} A_{j}
$$

The filtration $F$ is exhaustive and separated. Moreover, $A \cong \operatorname{gr}^{F} A$ as graded algebras. The isomorphism sends $a \in A_{i}$ to its symbol $\operatorname{symb}^{F}(a) \in \operatorname{gr}_{i}^{F} A$.

Lemma 1.1. Suppose that the $\mathbb{k}$-algebra $A$ is generated by a sequence of elements $\left\{a_{i}\right\}_{i \in I}$, where $I$ is an indexing set (possibly infinite). Given a sequence $\left\{d_{i}\right\}_{i \in I}$ of nonnegative integers, there is a unique nonnegative exhaustive filtration $F=\left\{F_{d} A\right\}_{d \in \mathbb{Z}}$ such that:

(i) for every $d, F_{d} A$ is the $\mathbb{k}$-linear span of the products $a_{j_{1}} \cdots a_{j_{m}}$ such that $d_{j_{1}}+\cdots+d_{j_{m}} \leqslant d$;

(ii) the graded algebra $\operatorname{gr}^{F} A$ is generated by a sequence of elements $\left\{\bar{a}_{i}\right\}_{i \in I}$, where for every $i \in I$ either $\bar{a}_{i}=\operatorname{symb}^{F}\left(a_{i}\right) \in \operatorname{gr}_{d_{i}}^{F} A$ or $\bar{a}_{i}=0$.

Proof. Let $\boldsymbol{x}=\left\{x_{i}\right\}_{i \in I}$ be a sequence of distinct indeterminates, and let $\mathbb{k}\langle\boldsymbol{x}\rangle$ be the free associative algebra on these generators. Define $\phi: \mathbb{k}\langle\boldsymbol{x}\rangle \rightarrow A$ to be the surjection sending $x_{i} \mapsto a_{i}$. Put on $\mathbb{k}\langle\boldsymbol{x}\rangle$ the grading such that $\operatorname{deg}\left(x_{i}\right)=d_{i}$. This induces a filtration $F=\left\{F_{d} \mathbb{K}\langle\boldsymbol{x}\rangle\right\}_{d \in \mathbb{Z}}$ where

$$
F_{d} \mathbb{k}\langle\boldsymbol{x}\rangle:=\bigoplus_{e \leqslant d} \mathbb{k}\langle\boldsymbol{x}\rangle_{e}
$$

This filtration can now be transferred to $A$ by setting $F_{d} A:=\phi\left(F_{d} \mathbb{k}\langle\boldsymbol{x}\rangle\right)$. Clearly $(A, F)$ is exhaustive and nonnegative, and also condition (i) holds. This condition also guarantees uniqueness.

As for condition (ii), consider the surjective graded algebra homomorphism

$$
\operatorname{gr}^{F}(\phi): \operatorname{gr}^{F} \mathbb{k}\langle\boldsymbol{x}\rangle \rightarrow \operatorname{gr}^{F} A .
$$

Because of the way the filtration on $\mathbb{k}\langle\boldsymbol{x}\rangle$ was constructed, the graded algebra $\operatorname{gr}^{F} \mathbb{k}\langle\boldsymbol{x}\rangle$ is a free algebra on the symbols $\bar{x}_{i}:=\operatorname{symb}^{F}\left(x_{i}\right)$. Define $\bar{a}_{i}:=\operatorname{gr}^{F}(\phi)\left(\bar{x}_{i}\right)$. These elements have the required properties. 


\section{A. YeKutieli And J. J. Zhang}

Conversely, we have the following two lemmas, whose standard proofs we leave out.

Lemma 1.2. Let $F=\left\{F_{d} A\right\}$ be an exhaustive nonnegative filtration of $A$, and let $\left\{a_{i}\right\}_{i \in I}$ be a sequence in $A$. Denote by $\bar{a}_{i}:=\operatorname{symb}^{F}\left(a_{i}\right)$. Suppose that the sequence $\left\{\bar{a}_{i}\right\}_{i \in I}$ generates $\operatorname{gr}^{F} A$ as $\mathbb{k}$-algebra. Then:

(1) $A$ is generated by $\left\{a_{i}\right\}_{i \in I}$ as $\mathbb{k}$-algebra;

(2) let $d_{i}:=\max \left\{0, \operatorname{deg}^{F}\left(a_{i}\right)\right\}$. Then $F$ coincides with the filtration from Lemma 1.1.

Lemma 1.3. Let $(A, F)$ be a nonnegative exhaustive filtered $\mathbb{k}$-algebra and let $(M, F)$ be a bounded below exhaustive filtered $(A, F)$-module. Suppose that $\left\{a_{i}\right\}_{i \in I} \subset A,\left\{b_{j}\right\}_{j \in J} \subset A$ and $\left\{c_{k}\right\}_{k \in K} \subset M$ are sequences satisfying:

(i) the set of symbols $\left\{\bar{a}_{i}\right\}_{i \in I} \cup\left\{\bar{b}_{j}\right\}_{j \in J}$ generates gr ${ }^{F} A$ as $\mathbb{k}$-algebra;

(ii) the set of symbols $\left\{\bar{c}_{k}\right\}_{k \in K}$ generates $\operatorname{gr}^{F} M$ as $\left(\operatorname{gr}^{F} A\right)$-module;

(iii) for every $i, j$ the symbols $\bar{a}_{i}$ and $\bar{b}_{j}$ commute.

Then for every integer $d$ the $\mathbb{k}$-module $F_{d} M$ is generated by the set of products

$$
\left\{a_{i_{1}} \cdots a_{i_{p}} b_{j_{1}} \cdots b_{j_{q}} c_{k} \mid \operatorname{deg}^{F}\left(a_{i_{1}}\right)+\cdots+\operatorname{deg}^{F}\left(b_{j_{1}}\right)+\cdots+\operatorname{deg}^{F}\left(c_{k}\right) \leqslant d\right\} .
$$

The base field $\mathbb{k}$ is of course trivially filtered. The filtered $\mathbb{k}$-modules $(M, F)$ form an additive category FiltMod $\mathbb{k}$, in which a morphism $\phi:(M, F) \rightarrow(N, F)$ is a $\mathbb{k}$-linear homomorphism $\phi: M \rightarrow$ $N$ such that $\phi\left(F_{i} M\right) \subset F_{i} N$.

The Rees module of $(M, F)$ is

$$
\operatorname{Rees}(M, F)=\operatorname{Rees}^{F} M:=\bigoplus_{i \in \mathbb{Z}} F_{i} M \cdot t^{i} \subset M[t]=M \otimes_{\mathbb{k}} \mathbb{k}[t]
$$

where $t$ is a central indeterminate of degree 1 . We get an additive functor

$$
\text { Rees : FiltMod } \mathbb{k} \rightarrow \text { GrMod } \mathbb{k}[t]
$$

where $\operatorname{GrMod} \mathbb{k}[t]$ is the abelian category of graded $\mathbb{k}[t]$-modules and degree 0 homomorphisms.

For a scalar $\lambda \in \mathbb{k}$ let us denote by $\operatorname{sp}_{\lambda}$ the specialization to $\lambda$ of a $\mathbb{k}[t]$-module $\widetilde{M}$, namely

$$
\operatorname{sp}_{\lambda} \widetilde{M}:=\widetilde{M} /(t-\lambda) \widetilde{M} \text {. }
$$

If $\lambda \neq 0$ then

$$
\mathrm{sp}_{\lambda}: \operatorname{GrMod} \mathbb{k}[t] \rightarrow \operatorname{Mod} \mathbb{k}
$$

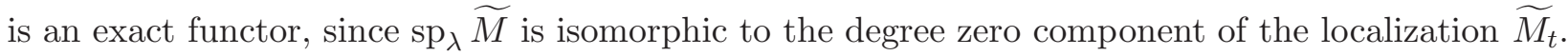
For $\lambda=0$ we get a functor

$$
\mathrm{sp}_{0}: \operatorname{GrMod} \mathbb{k}[t] \rightarrow \text { GrModk} .
$$

Given any $(M, F) \in$ FiltMod $\mathbb{k}$ one has

$$
\operatorname{sp}_{0} \operatorname{Rees}(M, F) \cong \operatorname{gr}(M, F)=\operatorname{gr}^{F} M .
$$

On the other hand, given a graded $\mathbb{k}[t]$-module $\widetilde{M}$ there is a filtration $F$ on $M:=\operatorname{sp}_{1} \widetilde{M}$ defined by

$$
F_{i} M:=\operatorname{Im}\left(\bigoplus_{j \leqslant i} \widetilde{M}_{j} \rightarrow M\right) .
$$

This is a functor

If $(M, F)$ is exhaustive, then

$$
\mathrm{sp}_{1}: \operatorname{GrMod} \mathbb{k}[t] \rightarrow \text { FiltModk }
$$

$$
\operatorname{sp}_{1} \operatorname{Rees}(M, F) \cong(M, F) .
$$




\section{DifFERENTIAL ALGEBRAS}

For a graded module $\widetilde{M} \in \operatorname{GrMod} \mathbb{k}[t]$ we have

$$
\text { Rees } \operatorname{sp}_{1} \widetilde{M} \cong \widetilde{M} /\{t \text {-torsion }\} .
$$

If $A$ is a filtered $\mathbb{k}$-algebra, then $\widetilde{A}:=\operatorname{Rees} A$ and $\bar{A}:=\operatorname{gr} A$ are graded algebras, and we obtain corresponding functors Rees, $\mathrm{sp}_{1}$ and $\mathrm{sp}_{0}$ between FiltMod $A, \operatorname{GrMod} \widetilde{A}$ and GrMod $\bar{A}$.

The next lemma states that a filtration can be lifted to the Rees ring.

Lemma 1.4. Let $F$ be an exhaustive nonnegative filtration of the $\mathbb{k}$-algebra $A$, and let $\widetilde{A}:=$ $\operatorname{Rees}^{F} A \subset A[t]$. For any integer $i$ define

Then:

$$
\widetilde{F}_{i} \widetilde{A}:=\bigoplus_{j \in \mathbb{N}}\left(F_{\min (i, j)} A\right) \cdot t^{j} \in A[t] .
$$

(i) $\widetilde{A}=\bigcup_{i} \widetilde{F}_{i} \widetilde{A}, \widetilde{F}_{-1} \widetilde{A}=0$ and $\widetilde{F}_{i} \widetilde{A} \cdot \widetilde{F}_{j} \widetilde{A} \subset \widetilde{F}_{i+j} \widetilde{A}$, thus $\widetilde{F}=\left\{\widetilde{F}_{i} \widetilde{A}\right\}$ is an exhaustive nonnegative filtration of the algebra $\widetilde{A}$;

(ii) there is an isomorphism of $\mathbb{k}$-algebras

$$
\operatorname{gr}^{\widetilde{F}} \widetilde{A} \cong\left(\operatorname{gr}^{F} A\right) \otimes \mathbb{k}[t]
$$

(not respecting degrees).

Proof. (1) Since $F_{-1} A=0$, we get $\widetilde{F}_{-1} \widetilde{A}=0$. Let $\widetilde{A}_{j}:=\left(F_{j} A\right) \cdot t^{j}$, the $j$ th graded component of $\widetilde{A}$. Then for any $i, j$ there is equality

$$
\widetilde{A}_{j} \cap\left(\widetilde{F}_{i} \widetilde{A}\right)=\left(F_{\min (i, j)} A\right) \cdot t^{j} .
$$

Hence $\widetilde{A}_{j} \subset \widetilde{F}_{j} \widetilde{A}$. It remains to check the products. For any two pairs of numbers $(i, k)$ and $(j, l)$ one has

$$
\min (i, k)+\min (j, l) \leqslant \min (i+j, k+l) .
$$

Therefore

$$
\left(\left(F_{\min (i, k)} A\right) \cdot t^{k}\right) \cdot\left(\left(F_{\min (j, l)} A\right) \cdot t^{l}\right) \subset\left(\left(F_{\min (i+j, k+l)} A\right) \cdot t^{k+l}\right) .
$$

This says that $\widetilde{F}_{i} \widetilde{A} \cdot \widetilde{F}_{j} \widetilde{A} \subset \widetilde{F}_{i+j} \widetilde{A}$.

(2) We have isomorphisms

$$
\operatorname{gr}^{\widetilde{F}} \widetilde{A} \cong \bigoplus_{0 \leqslant i} \bigoplus_{i \leqslant j}\left(\operatorname{gr}_{i}^{F} A\right) \cdot t^{j}
$$

and

$$
\left(\operatorname{gr}^{F} A\right) \otimes \mathbb{k}[t] \cong \bigoplus_{0 \leqslant i} \bigoplus_{0 \leqslant j}\left(\operatorname{gr}_{i}^{F} A\right) \cdot t^{j}
$$

The isomorphism $\operatorname{gr}^{\widetilde{F}} \widetilde{A} \stackrel{\simeq}{\longrightarrow}\left(\operatorname{gr}^{F} A\right) \otimes \mathbb{k}[t]$ we want is defined on every summand $\left(\operatorname{gr}_{i}^{F} A\right) \cdot t^{j}$ by dividing by $t^{i}$.

\section{Differential $\mathbb{k}$-algebras of finite type}

Let $C$ be a ring. Recall that a $C$-ring is a ring $A$ together with a ring homomorphism $C \rightarrow A$, called the structural homomorphism. Observe that a $C$-ring is also a $C$-bimodule.

Definition 2.1. Suppose $C$ is a commutative $\mathbb{k}$-algebra and $A$ is a $C$-ring. A differential $C$-filtration on $A$ is a filtration $F=\left\{F_{i} A\right\}_{i \in \mathbb{Z}}$ with the following properties:

(i) $1 \in F_{0} A$ and $F_{i} A \cdot F_{j} A \subset F_{i+j} A$;

(ii) $F_{-1} A=0$ and $A=\bigcup F_{i} A$; 


\section{A. YeKutieli And J. J. Zhang}

(iii) each $F_{i} A$ is a $C$-sub-bimodule;

(iv) the graded ring $\operatorname{gr}^{F} A$ is a $C$-algebra.

$A$ is called a differential $C$-ring if it admits some differential $C$-filtration.

The name 'differential filtration' signifies the similarity to Grothendieck's definition of differential operators; see [EG67].

Note that properties (i) and (iii) imply that the image of the structural homomorphism $C \rightarrow A$ lies in $F_{0} A$, so that (iv) makes sense.

Definition 2.2. Let $C$ be a commutative noetherian $\mathbb{k}$-algebra and $A$ a $C$-ring. A differential $C$-filtration of finite type on $A$ is a differential $C$-filtration $F=\left\{F_{i} A\right\}$ such that the graded $C$-algebra $\operatorname{gr}^{F} A$ is a finite module over its center $\mathrm{Z}\left(\operatorname{gr}^{F} A\right)$, and $\mathrm{Z}\left(\operatorname{gr}^{F} A\right)$ is a finitely generated $C$-algebra. We say that $A$ is a differential $C$-ring of finite type if it admits some differential $C$-filtration of finite type.

If $C$ is a finitely generated commutative $\mathbb{k}$-algebra and $A$ is a differential $C$-ring of finite type, then $A$ is also a differential $\mathbb{k}$-ring of finite type. In this case we also call $A$ a differential $\mathbb{k}$-algebra of finite type.

Example 2.3. Let $C$ be a finitely generated commutative $\mathbb{k}$-algebra and $A$ a finite $C$-algebra. Then $A$ is a differential $C$-ring of finite type. As filtration we can take the trivial filtration $F_{-1} A:=0$ and $F_{0} A:=A$.

Example 2.4. Suppose char $\mathbb{k}=0, C$ is a smooth commutative $\mathbb{k}$-algebra and $A:=\mathcal{D}(C)$ is the ring of $\mathbb{k}$-linear differential operators. Then $A$ is a differential $C$-ring of finite type. For filtration we can take the filtration $F=\left\{F_{i} A\right\}$ by order of operator, in which $F_{-1} A:=0, F_{0} A:=C, F_{1} A:=C \oplus \mathcal{T}(C)$ and $F_{i+1} A:=F_{i} A \cdot F_{1} A$ for $i \geqslant 1$. Here $\mathcal{T}(C):=\operatorname{Der}_{\mathbb{k}}(C)$, the module of derivations.

Example 2.5. A special case of Example 2.4 is when $C:=\mathbb{k}\left[x_{1}, \ldots, x_{n}\right]$, a polynomial ring. Then $A$ is called the $n$th Weyl algebra. Writing $y_{i}:=\partial / \partial x_{i}$ the algebra $A$ is generated by the $2 n$ elements $x_{1}, \ldots, x_{n}, y_{1}, \ldots, y_{n}$, with relations $\left[x_{i}, x_{j}\right]=\left[y_{i}, y_{j}\right]=\left[y_{i}, x_{j}\right]=0$ for $i \neq j$ and $\left[y_{i}, x_{i}\right]=1$. In addition to the filtration $F$ above there is also a differential $\mathbb{k}$-filtration of finite type $G=\left\{G_{i} A\right\}$ where $G_{-1} A:=0, G_{0} A:=\mathbb{k}, G_{1} A:=\mathbb{k}+\left(\sum_{i} \mathbb{k} \cdot x_{i}\right)+\left(\sum_{i} \mathbb{k} \cdot y_{i}\right)$ and $G_{i+1} A:=G_{i} A \cdot G_{1} A$ for $i \geqslant 1$.

Example 2.6. This example generalizes Example 2.5. Let $C$ be a finitely generated commutative $\mathbb{k}$-algebra (not necessarily smooth, and char $\mathbb{k}$ arbitrary), and let $L$ be a finite $C$-module (not necessarily projective). Suppose $L$ has a $\mathbb{k}$-linear Lie bracket $[-,-]$. The module of derivations $\mathcal{T}(C):=\operatorname{Der}_{\mathbb{k}}(C)$ is also a $\mathbb{k}$-Lie algebra. Suppose $\alpha: L \rightarrow \mathcal{T}(C)$ is a $C$-linear Lie homomorphism, namely $\alpha(c \xi)=c \alpha(\xi)$ and $\alpha([\xi, \zeta])=[\alpha(\xi), \alpha(\zeta)]$ for all $c \in C$ and $\xi, \zeta \in L$. L is then called a Lie algebroid or a Lie-Rinehart algebra (cf. [Ch99, Ri63]). The ring of generalized differential operators $\mathcal{D}(C ; L)$, also called the universal enveloping algebra and denoted by $\mathrm{U}(C ; L)$, is defined as follows. Choose $\mathbb{k}$-algebra generators $c_{1}, \ldots, c_{p}$ for $C$ and $C$-module generators $l_{1}, \ldots, l_{q}$ for $L$. Let

$$
\mathbb{k}\langle\boldsymbol{x}, \boldsymbol{y}\rangle:=\mathbb{k}\left\langle x_{1}, \ldots, x_{p}, y_{1}, \ldots, y_{q}\right\rangle
$$

be the free associative algebra. We have a ring surjection $\phi_{0}: \mathbb{k}\langle\boldsymbol{x}\rangle \rightarrow C$ with $\phi_{0}\left(x_{i}\right):=c_{i}$. Let $I_{0}:=\operatorname{Ker}\left(\phi_{0}\right)$. Next there is a surjection of $\mathbb{k}\langle\boldsymbol{x}\rangle$-modules

$$
\phi_{1}: \mathbb{k}\langle\boldsymbol{x}\rangle^{q}=\bigoplus_{j=1}^{q} \mathbb{k}\langle\boldsymbol{x}\rangle \cdot y_{j} \rightarrow L
$$

with $\phi_{1}\left(y_{j}\right):=l_{j}$. Define $I_{1}:=\operatorname{Ker}\left(\phi_{1}\right) \subset \mathbb{k}\langle\boldsymbol{x}\rangle^{q}$. For any $i, j$ choose polynomials $f_{i, j}(\boldsymbol{x})$ and $g_{i, j, k}(\boldsymbol{x})$ such that $\left[l_{i}, l_{j}\right]=\sum_{k} g_{i, j, k}(\boldsymbol{c}) l_{k} \in L$ and $\alpha\left(l_{i}\right)\left(c_{j}\right)=f_{i, j}(\boldsymbol{c}) \in C$. Now define

$$
\mathrm{U}(C ; L):=\frac{\mathbb{k}\langle\boldsymbol{x}, \boldsymbol{y}\rangle}{I}
$$




\section{DifFERENTIAL ALGEBRAS}

where $I \subset \mathbb{k}\langle\boldsymbol{x}, \boldsymbol{y}\rangle$ is the two-sided ideal generated by $I_{0}, I_{1}$ and the polynomials $\left[y_{i}, y_{j}\right]-$ $\sum_{k} g_{i, j, k}(\boldsymbol{x}) y_{k}$ and $\left[y_{i}, x_{j}\right]-f_{i, j}(\boldsymbol{x})$.

The ring $\mathrm{U}(C ; L)$ has the following universal property: given any ring $D$, any ring homomorphism $\eta_{0}: C \rightarrow D$ and any $C$-linear Lie homomorphism $\eta_{1}: L \rightarrow D$ satisfying $\left[\eta_{1}(l), \eta_{0}(c)\right]=\eta_{0}(\alpha(l)(c))$, there is a unique ring homomorphism $\eta: \mathrm{U}(C ; L) \rightarrow D$ through which $\eta_{0}$ and $\eta_{1}$ factor.

Put on $\mathbb{k}\langle\boldsymbol{x}, \boldsymbol{y}\rangle$ the filtration $F$ such that $\operatorname{deg}^{F}\left(x_{i}\right)=0$ and $\operatorname{deg}^{F}\left(y_{j}\right)=1$. Let $F$ be the filtration induced on $\mathrm{U}(C ; L)$ by the surjection $\phi: \mathbb{k}\langle\boldsymbol{x}, \boldsymbol{y}\rangle \rightarrow \mathrm{U}(C ; L)$. Then $\operatorname{gr}^{F} \mathrm{U}(C ; L)$ is a commutative $C$-algebra, generated by the elements $\bar{l}_{j}:=\operatorname{gr}^{F}(\phi)\left(y_{j}\right), j \in\{1, \ldots, q\}$. We see that $\mathrm{U}(C ; L)$ is a differential $C$-ring of finite type. If $C=\mathbb{k}\left[x_{1}, \ldots, x_{n}\right]$ and $L=\mathcal{T}(C)$, then we are in the situation of Example 2.5. If $C=\mathbb{k}$, then $\mathrm{U}(C ; L)=\mathrm{U}(L)$ is the usual universal enveloping algebra of the Lie algebra $L$.

Lemma 2.7 [ATV91, Theorem 8.2]. Suppose that $A=\bigoplus_{i \in \mathbb{N}} A_{i}$ is a graded $\mathbb{k}$-algebra and $t \in A$ is a central homogeneous element of positive degree. The following are equivalent:

(i) $A$ is left noetherian;

(ii) $A /(t)$ is left noetherian.

The next proposition follows almost directly from the definition and Lemma 2.7.

Proposition 2.8. If $A$ is a differential $\mathbb{k}$-algebra of finite type, then it is a noetherian finitely generated $\mathbb{k}$-algebra.

The class of differential $\mathbb{k}$-algebras of finite type is closed under tensor products as we now show.

Proposition 2.9. Let $C_{1}$ and $C_{2}$ be noetherian commutative $\mathbb{k}$-algebras, and assume that $C_{1} \otimes C_{2}$ is also noetherian. Let $A_{i}$ be a differential $C_{i}$-ring of finite type for $i=1,2$. Then $A_{1} \otimes A_{2}$ is a differential $\left(C_{1} \otimes C_{2}\right)$-ring of finite type.

Proof. Choose differential filtrations of finite type $\left\{F_{n} A_{1}\right\}$ and $\left\{F_{n} A_{2}\right\}$ of $A_{1}$ and $A_{2}$. Define a filtration on $A_{1} \otimes A_{2}$ as follows:

$$
F_{n}\left(A_{1} \otimes A_{2}\right):=\sum_{l+m=n} F_{l} A_{1} \otimes F_{m} A_{2} .
$$

Then

$$
\operatorname{gr}^{F}\left(A_{1} \otimes A_{2}\right) \cong\left(\operatorname{gr}^{F} A_{1}\right) \otimes\left(\operatorname{gr}^{F} A_{2}\right)
$$

as graded rings. Since $\operatorname{gr}^{F} A_{1}$ and $\operatorname{gr}^{F} A_{2}$ are finite modules over their centers it follows that $\left(\operatorname{gr}^{F} A_{1}\right) \otimes$ $\left(\mathrm{gr}^{F} A_{2}\right)$ is a finite module over its center.

\section{The theorem on the two filtrations}

The next theorem generalizes the case of the $n$th Weyl algebra and its two filtrations (see Examples 2.4 and 2.5 above). McConnell and Stafford also considered such filtrations, and our result extends their [MS89, Corollary 1.7]. The basic idea is attributed in [MS89] to Bernstein.

We recall that a graded $\mathbb{k}$-algebra $A$ is called connected if $A=\bigoplus_{i \in \mathbb{N}} A_{i}, A_{0}=\mathbb{k}$ and each $A_{i}$ is a finite $\mathbb{k}$-module.

Theorem 3.1. Let $A$ be a $\mathbb{k}$-algebra. Assume that $A$ has a differential $\mathbb{k}$-filtration of finite type $F=\left\{F_{i} A\right\}_{i \in \mathbb{Z}}$. Then there is a nonnegative exhaustive $\mathbb{k}$-filtration $G=\left\{G_{i} A\right\}_{i \in \mathbb{Z}}$ such that $\operatorname{gr}^{G} A$ is a commutative, finitely generated, connected graded $\mathbb{k}$-algebra. 


\section{A. YeKutieli And J. J. Zhang}

Observe that $G$ is also a differential filtration of finite type on $A$. As mentioned in the introduction, Theorem 3.1 is used to prove the existence of an Auslander rigid dualizing complex over $A$.

The following easy lemma is used often in the proof of the theorem.

Lemma 3.2. Let $F=\left\{F_{i} A\right\}$ be a nonnegative exhaustive filtration of $A$ and let $a_{1}, a_{2} \in A$ be two elements. Define $\bar{a}_{i}:=\operatorname{symb}^{F}\left(a_{i}\right) \in \operatorname{gr}^{F} A$ and $d_{i}:=\operatorname{deg}^{F}\left(a_{i}\right) \in \mathbb{N} \cup\{-\infty\}$. Then the commutator $\left[\bar{a}_{1}, \bar{a}_{2}\right]=0$ if and only if

$$
\operatorname{deg}^{F}\left(\left[a_{1}, a_{2}\right]\right) \leqslant d_{1}+d_{2}-1
$$

Proof of Theorem 3.1. Step 1. Write $\bar{A}:=\operatorname{gr}^{F} A$. Then the center $\mathrm{Z}(\bar{A})$ is a graded, finitely generated, commutative $\mathbb{k}$-algebra, and $\bar{A}$ is a finite $\mathrm{Z}(\bar{A})$-module. For any element $a \in A$ we write $\bar{a}:=\operatorname{symb}^{F}(a) \in \operatorname{gr}^{F} A$.

Let $d_{1} \in \mathbb{N}$ be large enough such that $\mathrm{Z}(\bar{A})$ is generated as $\mathrm{Z}(\bar{A})_{0}$-algebra by finitely many elements of degrees $\leqslant d_{1}$, and $\bar{A}$ is generated as $\mathrm{Z}(\bar{A})$-module by finitely many elements of degrees $\leqslant d_{1}$.

Choose nonzero elements $a_{1}, \ldots, a_{m} \in F_{0} A \cong \bar{A}_{0}$ such that their symbols $\bar{a}_{1}, \ldots, \bar{a}_{m}$ are in $\mathrm{Z}(\bar{A})_{0}$, and they generate $\mathrm{Z}(\bar{A})_{0}$ as $\mathbb{k}$-algebra. Next choose elements $b_{1}, \ldots, b_{n} \in F_{d_{1}} A-F_{0} A$ such that the symbols $\bar{b}_{1}, \ldots, \bar{b}_{n}$ are in $\mathrm{Z}(\bar{A})$, and they generate $\mathrm{Z}(\bar{A})$ as $\mathrm{Z}(\bar{A})_{0}$-algebra. Finally choose nonzero elements $c_{1}, \ldots, c_{p} \in F_{d_{1}} A$ such that the symbols $\bar{c}_{1}, \ldots, \bar{c}_{p}$ generate $\bigoplus_{j=0}^{d_{1}} \bar{A}_{j}$ as $\mathrm{Z}(\bar{A})_{0}$-module. This implies that $\bar{c}_{1}, \ldots, \bar{c}_{p}$ generate $\bar{A}$ as a $\mathrm{Z}(\bar{A})$-module.

The symbols $\bar{a}_{1}, \ldots, \bar{a}_{m}, \bar{b}_{1}, \ldots, \bar{b}_{n}, \bar{c}_{1}, \ldots, \bar{c}_{p}$ generate $\bar{A}$ as $\mathbb{k}$-algebra, so by Lemma 1.2 the elements $a_{1}, \ldots, c_{m}, b_{1}, \ldots, b_{n}, c_{1}, \ldots, c_{p}$ generate $A$ as $\mathbb{k}$-algebra. Let

$$
\mathbb{k}\langle\boldsymbol{x}, \boldsymbol{y}, \boldsymbol{z}\rangle:=\left\langle x_{1}, \ldots, x_{m}, y_{1}, \ldots, y_{n}, z_{1}, \ldots, z_{p}\right\rangle
$$

be the free associative algebra, and define a surjective ring homomorphism $\phi: \mathbb{k}\langle\boldsymbol{x}, \boldsymbol{y}, \boldsymbol{z}\rangle \rightarrow A$ by sending $x_{i} \mapsto a_{i}, y_{i} \mapsto b_{i}$ and $z_{i} \mapsto c_{i}$. We are now in the situation of Lemma 1.2. The free algebra $\mathbb{k}\langle\boldsymbol{x}, \boldsymbol{y}, \boldsymbol{z}\rangle$ also has a filtration $F$, where $\operatorname{deg}^{F}\left(x_{i}\right):=\operatorname{deg}^{F}\left(a_{i}\right), \operatorname{deg}^{F}\left(y_{i}\right):=\operatorname{deg}^{F}\left(b_{i}\right)$ etc., and $\phi$ is a strict surjection, meaning that $F_{i}(A)=\phi\left(F_{i} \mathbb{k}\langle\boldsymbol{x}, \boldsymbol{y}, \boldsymbol{z}\rangle\right)$.

Let us denote substitution by $f(\boldsymbol{a}, \boldsymbol{b}, \boldsymbol{c}):=\phi(f(\boldsymbol{x}, \boldsymbol{y}, \boldsymbol{z}))$. Consider the subrings

$$
\mathbb{k}\langle\boldsymbol{a}\rangle \subset \mathbb{k}\langle\boldsymbol{a}, \boldsymbol{b}\rangle \subset A=\mathbb{k}\langle\boldsymbol{a}, \boldsymbol{b}, \boldsymbol{c}\rangle
$$

with filtrations $F$ induced by the inclusions into $A$. The reader is warned that these filtrations might differ from the filtrations induced by $\phi: \mathbb{k}\langle\boldsymbol{x}\rangle \rightarrow \mathbb{k}\langle\boldsymbol{a}\rangle$ and $\phi: \mathbb{k}\langle\boldsymbol{x}, \boldsymbol{y}\rangle \rightarrow \mathbb{k}\langle\boldsymbol{a}, \boldsymbol{b}\rangle$ respectively.

We observe that the commutators $\left[a_{i}, a_{j}\right]=0$ for all $i, j$, since $a_{i}=\bar{a}_{i} \in Z(\bar{A})_{0}$. This also says that $\left[\bar{a}_{i}, \bar{b}_{j}\right]=0$, so according to Lemma 3.2 we get

$$
\left[a_{i}, b_{j}\right] \in F_{\operatorname{deg}^{F}\left(b_{j}\right)-1} A
$$

for all $i, j$. Therefore, by Lemma 1.3 , applied to the filtered $\mathbb{k}$-algebra $\mathbb{k}\langle\boldsymbol{a}\rangle$ and the filtered $\mathbb{k}\langle\boldsymbol{a}\rangle$-module $F_{d_{1}} A$, we see that there are noncommutative polynomials $f_{i, j, k}^{1}(\boldsymbol{x}) \in \mathbb{k}\langle\boldsymbol{x}\rangle$ such that

$$
\operatorname{deg}^{F}\left(f_{i, j, k}^{1}(\boldsymbol{x})\right)+\operatorname{deg}^{F}\left(c_{k}\right) \leqslant \operatorname{deg}^{F}\left(b_{j}\right)-1 \quad \text { and } \quad\left[a_{i}, b_{j}\right]=\sum_{k} f_{i, j, k}^{1}(\boldsymbol{a}) \cdot c_{k} .
$$

Note that either $f_{i, j, k}^{1}(\boldsymbol{x}) \neq 0$, in which case $\operatorname{deg}^{F}\left(f_{i, j, k}^{1}(\boldsymbol{x})\right)=0$; or $f_{i, j, k}^{1}(\boldsymbol{x})=0$ and then $\operatorname{deg}^{F}\left(f_{i, j, k}^{1}(\boldsymbol{x})\right)=-\infty$. The choice $f_{i, j, k}^{1}(\boldsymbol{x})=0$ is of course required when $\operatorname{deg}^{F}\left(c_{k}\right) \geqslant \operatorname{deg}^{F}\left(b_{j}\right)$.

Likewise $\left[b_{i}, b_{j}\right] \in F_{\operatorname{deg}^{F}\left(b_{i}\right)+\operatorname{deg}^{F}\left(b_{j}\right)-1} A$, so by Lemma 1.3, applied to the filtered $\mathbb{k}$-algebra $\mathbb{k}\langle\boldsymbol{a}, \boldsymbol{b}\rangle$ and the filtered $\mathbb{k}\langle\boldsymbol{a}, \boldsymbol{b}\rangle$-module $A$, we see that there are noncommutative polynomials $f_{i, j, k}^{2}(\boldsymbol{x})$ 


\section{DifFERENTIAL ALGEBRAS}

and $g_{i, j, k}^{2}(\boldsymbol{y})$ such that

$$
\begin{gathered}
\operatorname{deg}^{F}\left(f_{i, j, k}^{2}(\boldsymbol{x})\right)+\operatorname{deg}^{F}\left(g_{i, j, k}^{2}(\boldsymbol{y})\right)+\operatorname{deg}^{F}\left(c_{k}\right) \leqslant \operatorname{deg}^{F}\left(b_{i}\right)+\operatorname{deg}^{F}\left(b_{j}\right)-1 \\
\text { and }\left[b_{i}, b_{j}\right]=\sum_{k} f_{i, j, k}^{2}(\boldsymbol{a}) \cdot g_{i, j, k}^{2}(\boldsymbol{b}) \cdot c_{k} .
\end{gathered}
$$

Similarly there are polynomials $f_{i, j, k}^{3}(\boldsymbol{x})$ such that

$$
\operatorname{deg}^{F}\left(f_{i, j, k}^{3}(\boldsymbol{x})\right)+\operatorname{deg}^{F}\left(c_{k}\right) \leqslant \operatorname{deg}^{F}\left(c_{j}\right)-1 \quad \text { and } \quad\left[a_{i}, c_{j}\right]=\sum_{k} f_{i, j, k}^{3}(\boldsymbol{a}) \cdot c_{k},
$$

and there are polynomials $f_{i, j, k}^{4}(\boldsymbol{x})$ and $g_{i, j, k}^{4}(\boldsymbol{y})$ such that

$$
\begin{gathered}
\operatorname{deg}^{F}\left(f_{i, j, k}^{4}(\boldsymbol{x})\right)+\operatorname{deg}^{F}\left(g_{i, j, k}^{4}(\boldsymbol{y})\right)+\operatorname{deg}^{F}\left(c_{k}\right) \leqslant \operatorname{deg}^{F}\left(b_{i}\right)+\operatorname{deg}^{F}\left(c_{j}\right)-1 \\
\text { and }\left[b_{i}, c_{j}\right]=\sum_{k} f_{i, j, k}^{4}(\boldsymbol{a}) \cdot g_{i, j, k}^{4}(\boldsymbol{b}) \cdot c_{k} .
\end{gathered}
$$

The same idea applies to $c_{i} c_{j}$ : there are polynomials $f_{i, j, k}^{5}(\boldsymbol{x})$ and $g_{i, j, k}^{5}(\boldsymbol{y})$ such that

$$
\begin{gathered}
\operatorname{deg}^{F}\left(f_{i, j, k}^{5}(\boldsymbol{x})\right)+\operatorname{deg}^{F}\left(g_{i, j, k}^{5}(\boldsymbol{y})\right)+\operatorname{deg}^{F}\left(c_{k}\right) \leqslant \operatorname{deg}^{F}\left(c_{i}\right)+\operatorname{deg}^{F}\left(c_{j}\right) \\
\text { and } c_{i} \cdot c_{j}=\sum_{k} f_{i, j, k}^{5}(\boldsymbol{a}) \cdot g_{i, j, k}^{5}(\boldsymbol{b}) \cdot c_{k} .
\end{gathered}
$$

Let $G$ be the standard grading on $\mathbb{k}\langle\boldsymbol{x}\rangle$, namely $\operatorname{deg}^{G}\left(x_{i}\right):=1$. This induces a filtration $G$. Define

$$
e_{0}:=\max \left\{0, \operatorname{deg}^{G}\left(f_{i, j, k}^{l}(\boldsymbol{x})\right)\right\},
$$

$e_{1}:=e_{0}+1$ and $e_{2}:=e_{0}+e_{1}+1$.

Put on the free algebra $\mathbb{k}\langle\boldsymbol{x}, \boldsymbol{y}, \boldsymbol{z}\rangle$ a new grading $G$ by declaring

$$
\begin{gathered}
\operatorname{deg}^{G}\left(y_{i}\right):=e_{2} \operatorname{deg}^{F}\left(b_{i}\right) \\
\operatorname{deg}^{G}\left(z_{i}\right):=e_{2} \operatorname{deg}^{F}\left(c_{i}\right)+e_{1},
\end{gathered}
$$

and keeping $\operatorname{deg}^{G}\left(x_{i}\right)=1$ as above. We get a new filtration $G$ on $\mathbb{k}\langle\boldsymbol{x}, \boldsymbol{y}, \boldsymbol{z}\rangle$. Using this we obtain a new filtration $G$ on $A$ with

$$
G_{i} A:=\phi\left(G_{i} \mathbb{k}\langle\boldsymbol{x}, \boldsymbol{y}, \boldsymbol{z}\rangle\right) .
$$

Step 2. Now we verify that the filtration $G$ has the required properties. Since the filtration $G$ on $\mathbb{k}\langle\boldsymbol{x}, \boldsymbol{y}, \boldsymbol{z}\rangle$ is nonnegative exhaustive, and $\phi$ is a strict surjection, it follows that the filtration $G$ on $A$ is also nonnegative exhaustive. The rest requires some work, and in order to simplify our notation we are going to 'recycle' the expressions $\bar{A}, \bar{a}_{i}$, etc. From here on we define $\bar{A}:=\operatorname{gr}^{G} A$. We have a surjective graded $\mathbb{k}$-algebra homomorphism

$$
\bar{\phi}:=\operatorname{gr}^{G}(\phi): \operatorname{gr}^{G} \mathbb{k}\langle\boldsymbol{x}, \boldsymbol{y}, \boldsymbol{z}\rangle \rightarrow \operatorname{gr}^{G} A=\bar{A} .
$$

Let $\bar{x}_{i}:=\operatorname{symb}^{G}\left(x_{i}\right), \bar{y}_{i}:=\operatorname{symb}^{G}\left(y_{i}\right)$, etc. Then

$$
\operatorname{gr}^{G} \mathbb{k}\langle\boldsymbol{x}, \boldsymbol{y}, \boldsymbol{z}\rangle=\mathbb{k}\langle\overline{\boldsymbol{x}}, \overline{\boldsymbol{y}}, \overline{\boldsymbol{z}}\rangle:=\mathbb{k}\left\langle\bar{x}_{1}, \ldots, \bar{x}_{m}, \bar{y}_{1}, \ldots, \bar{y}_{n}, \bar{z}_{1}, \ldots, \bar{z}_{p}\right\rangle
$$

which is also a free algebra. Define $\bar{a}_{i}:=\bar{\phi}\left(\bar{x}_{i}\right), \bar{b}_{i}:=\bar{\phi}\left(\bar{y}_{i}\right)$ and $\bar{c}_{i}:=\bar{\phi}\left(\bar{z}_{i}\right)$. Observe that either $\operatorname{deg}^{G}\left(a_{i}\right)=\operatorname{deg}^{G}\left(x_{i}\right)$, in which case $\bar{a}_{i}=\operatorname{symb}^{G}\left(a_{i}\right)$, and it is a nonzero element of $\bar{A}_{\operatorname{deg}^{G}\left(a_{i}\right)}$; or $\operatorname{deg}^{G}\left(a_{i}\right)<\operatorname{deg}^{G}\left(x_{i}\right)$, and then $\bar{a}_{i}=0$. Similar statements hold for $\bar{b}_{i}$ and $\bar{c}_{i}$.

Since $\bar{\phi}$ is surjective we see that $\bar{A}$ is generated as $\mathbb{k}$-algebra by the elements $\bar{a}_{1}, \ldots, \bar{a}_{m}, \bar{b}_{1}, \ldots, \bar{b}_{n}$, $\bar{c}_{1}, \ldots, \bar{c}_{p}$. These elements are either of positive degree or are 0 , and hence $\bar{A}$ is connected graded. We claim that $\bar{A}$ is commutative. 


\section{A. YeKutieli And J. J. Zhang}

We know already that $\left[\bar{a}_{i}, \bar{a}_{j}\right]=0$. Let us check that $\left[\bar{a}_{i}, \bar{b}_{j}\right]=0$. If either $\operatorname{deg}^{G}\left(a_{i}\right)<\operatorname{deg}^{G}\left(x_{i}\right)$ or $\operatorname{deg}^{G}\left(b_{j}\right)<\operatorname{deg}^{G}\left(y_{j}\right)$, then $\bar{a}_{i} \bar{b}_{j}=\bar{b}_{j} \bar{a}_{i}=0$. Otherwise, $\bar{a}_{i}=\operatorname{symb}^{G}\left(a_{i}\right)$ and $\bar{b}_{j}=\operatorname{symb}^{G}\left(b_{j}\right)$. By formula (3.3) we have

$$
\begin{aligned}
\operatorname{deg}^{G}\left(\left[a_{i}, b_{j}\right]\right) & \leqslant \max \left\{\operatorname{deg}^{G}\left(f_{i, j, k}^{1}(\boldsymbol{a})\right)+\operatorname{deg}^{G}\left(c_{k}\right)\right\} \\
& \leqslant \max \left\{\operatorname{deg}^{G}\left(f_{i, j, k}^{1}(\boldsymbol{x})\right)+\operatorname{deg}^{G}\left(c_{k}\right)\right\} .
\end{aligned}
$$

For any $k$ such that $f_{i, j, k}^{1}(\boldsymbol{x}) \neq 0$ we have $\operatorname{deg}^{F}\left(c_{k}\right) \leqslant \operatorname{deg}^{F}\left(b_{j}\right)-1$, and then

$$
\operatorname{deg}^{G}\left(c_{k}\right) \leqslant \operatorname{deg}^{G}\left(z_{k}\right)=e_{2} \operatorname{deg}^{F}\left(c_{k}\right)+e_{1} \leqslant e_{2}\left(\operatorname{deg}^{F}\left(b_{j}\right)-1\right)+e_{1} .
$$

Also $\operatorname{deg}^{G}\left(f_{i, j, k}^{1}(\boldsymbol{x})\right) \leqslant e_{0}$. Because

$$
\operatorname{deg}^{G}\left(b_{j}\right)=\operatorname{deg}^{G}\left(y_{j}\right)=e_{2} \operatorname{deg}^{F}\left(b_{j}\right)
$$

and

$$
\operatorname{deg}^{G}\left(a_{i}\right)=\operatorname{deg}^{G}\left(x_{i}\right)=1
$$

we get

$$
\begin{gathered}
\operatorname{deg}^{G}\left(\left[a_{i}, b_{j}\right]\right) \leqslant e_{0}+\left(e_{2}\left(\operatorname{deg}^{F}\left(b_{j}\right)-1\right)+e_{1}\right) \\
=\operatorname{deg}^{G}\left(a_{i}\right)+\operatorname{deg}^{G}\left(b_{j}\right)-2 .
\end{gathered}
$$

Using Lemma 3.2 we conclude that $\left[\bar{a}_{i}, \bar{b}_{j}\right]=0$.

Next let us consider the commutator $\left[\bar{b}_{i}, \bar{b}_{j}\right]$. If either $\operatorname{deg}^{G}\left(b_{i}\right)<\operatorname{deg}^{G}\left(y_{i}\right)$ or $\operatorname{deg}^{G}\left(b_{j}\right)<$ $\operatorname{deg}^{G}\left(y_{j}\right)$, then $\bar{b}_{i} \bar{b}_{j}=\bar{b}_{j} \bar{b}_{i}=0$. Otherwise, $\bar{b}_{i}=\operatorname{symb}^{G}\left(b_{i}\right)$ and $\bar{b}_{j}=\operatorname{symb}^{G}\left(b_{j}\right)$. By formula (3.4), if $f_{i, j, k}^{2}(\boldsymbol{x}) \neq 0$, then

$$
\operatorname{deg}^{F}\left(g_{i, j, k}^{2}(\boldsymbol{y})\right)+\operatorname{deg}^{F}\left(c_{k}\right) \leqslant \operatorname{deg}^{F}\left(b_{i}\right)+\operatorname{deg}^{F}\left(b_{j}\right)-1 .
$$

Also

$$
\begin{aligned}
\operatorname{deg}^{G}\left(g_{i, j, k}^{2}(\boldsymbol{y})\right) & =e_{2} \operatorname{deg}^{F}\left(g_{i, j, k}^{2}(\boldsymbol{y})\right) \\
\operatorname{deg}^{G}\left(c_{k}\right) & \leqslant \operatorname{deg}^{G}\left(z_{k}\right)=e_{2} \operatorname{deg}^{F}\left(c_{k}\right)+e_{1} .
\end{aligned}
$$

Therefore, looking only at indices $k$ such that $f_{i, j, k}^{2}(\boldsymbol{x}) \neq 0$, we obtain

$$
\begin{aligned}
\operatorname{deg}^{G}\left(\left[b_{i}, b_{j}\right]\right) & \leqslant \max \left\{\operatorname{deg}^{G}\left(f_{i, j, k}^{2}(\boldsymbol{a})\right)+\operatorname{deg}^{G}\left(g_{i, j, k}^{2}(\boldsymbol{b})\right)+\operatorname{deg}^{G}\left(c_{k}\right)\right\} \\
& \leqslant \max \left\{\operatorname{deg}^{G}\left(f_{i, j, k}^{2}(\boldsymbol{x})\right)+\operatorname{deg}^{G}\left(g_{i, j, k}^{2}(\boldsymbol{y})\right)+\operatorname{deg}^{G}\left(c_{k}\right)\right\} \\
& \leqslant e_{0}+\max \left\{e_{2} \operatorname{deg}^{F}\left(g_{i, j, k}^{2}(\boldsymbol{y})\right)+e_{2} \operatorname{deg}^{F}\left(c_{k}\right)+e_{1}\right\} \\
& =e_{2} \max \left\{\operatorname{deg}^{F}\left(g_{i, j, k}^{2}(\boldsymbol{y})\right)+\operatorname{deg}^{F}\left(c_{k}\right)+1\right\}+\left(e_{0}+e_{1}-e_{2}\right) \\
& \leqslant e_{2}\left(\operatorname{deg}^{F}\left(b_{i}\right)+\operatorname{deg}^{F}\left(b_{j}\right)\right)-1 \\
& =\operatorname{deg}^{G}\left(b_{i}\right)+\operatorname{deg}^{G}\left(b_{j}\right)-1 .
\end{aligned}
$$

So according to Lemma 3.2 we conclude that $\left[\bar{b}_{i}, \bar{b}_{j}\right]=0$.

The calculation for the other commutators is similar.

Finally we show that, amusingly, $\bar{c}_{i} \bar{c}_{j}=0$. If either $\operatorname{deg}^{G}\left(c_{i}\right)<\operatorname{deg}^{G}\left(z_{i}\right) \operatorname{or~deg}^{G}\left(c_{j}\right)<\operatorname{deg}^{G}\left(z_{j}\right)$, then automatically $\bar{c}_{i} \bar{c}_{j}=0$. Otherwise, $\bar{c}_{i}=\operatorname{symb}^{G}\left(c_{i}\right)$ and $\bar{c}_{j}=\operatorname{symb}^{G}\left(c_{j}\right)$. For any $k$ such that $f_{i, j, k}^{5}(\boldsymbol{x}) \neq 0$ one has

$$
\operatorname{deg}^{F}\left(g_{i, j, k}^{5}(\boldsymbol{y})\right)+\operatorname{deg}^{F}\left(c_{k}\right) \leqslant \operatorname{deg}^{F}\left(c_{i}\right)+\operatorname{deg}^{F}\left(c_{j}\right) .
$$




\section{DifFERENTIAL ALGEBRAS}

Therefore, looking only at indices $k$ such that $f_{i, j, k}^{5}(\boldsymbol{x}) \neq 0$, we obtain

$$
\begin{aligned}
\operatorname{deg}^{G}\left(c_{i} c_{j}\right) & \leqslant \max \left\{\operatorname{deg}^{G}\left(f_{i, j, k}^{5}(\boldsymbol{a})\right)+\operatorname{deg}^{G}\left(g_{i, j, k}^{5}(\boldsymbol{b})\right)+\operatorname{deg}^{G}\left(c_{k}\right)\right\} \\
& \leqslant \max \left\{\operatorname{deg}^{G}\left(f_{i, j, k}^{5}(\boldsymbol{x})\right)+\operatorname{deg}^{G}\left(g_{i, j, k}^{5}(\boldsymbol{y})\right)+\operatorname{deg}^{G}\left(c_{k}\right)\right\} \\
& \leqslant e_{0}+\max \left\{e_{2} \operatorname{deg}^{F}\left(g_{i, j, k}^{5}(\boldsymbol{y})\right)+e_{2} \operatorname{deg}^{F}\left(c_{k}\right)+e_{1}\right\} \\
& =e_{2} \max \left\{\operatorname{deg}^{F}\left(g_{i, j, k}^{5}(\boldsymbol{y})\right)+\operatorname{deg}^{F}\left(c_{k}\right)\right\}+\left(e_{0}+e_{1}\right) \\
& \leqslant e_{2}\left(\operatorname{deg}^{F}\left(c_{i}\right)+\operatorname{deg}^{F}\left(c_{j}\right)\right)+\left(e_{0}+e_{1}\right) \\
& =\operatorname{deg}^{G}\left(c_{i}\right)+\operatorname{deg}^{G}\left(c_{j}\right)-1 .
\end{aligned}
$$

So, by definition of the product in $\bar{A}$, we get $\bar{c}_{i} \bar{c}_{j}=0$.

Proposition 3.5. In the situation of Theorem 3.1 assume that the $\mathbb{k}$-algebra $A$ is graded, and that every $\mathbb{k}$-submodule $F_{i} A$ is also graded. Then the filtration $G$ can be chosen such that every $\mathbb{k}$-submodule $G_{i} A$ is graded.

Proof. Simply choose the generators $a_{1}, \ldots, b_{1}, \ldots, c_{1}, \ldots, c_{p} \in A$ used in the proof to be homogeneous.

\section{Review of dualizing complexes}

For a $\mathbb{k}$-algebra $A$ we denote by $A^{\mathrm{op}}$ the opposite algebra, and by $A^{\mathrm{e}}:=A \otimes A^{\text {op }}$ the enveloping algebra. Recall that an $A$-module means a left $A$-module. With this convention a right $A$-module is an $A^{\mathrm{op}}$-module, and an $A$-bimodule is an $A^{\mathrm{e}}$-module.

In this section we review the definition of dualizing complexes over rings and related concepts.

Let $\operatorname{Mod} A$ be the category of $A$-modules, and let $\operatorname{Mod}_{\mathrm{f}} A$ be the full subcategory of finite (i.e. finitely generated) modules. The latter is abelian when $A$ is left noetherian. Let $\mathrm{D}(\operatorname{Mod} A)$ be the derived category of $A$-modules. The full subcategory of bounded complexes is denoted by $\mathrm{D}^{\mathrm{b}}(\operatorname{Mod} A)$, the full subcategory of complexes with finite cohomologies is denoted by $\mathrm{D}_{\mathrm{f}}(\operatorname{Mod} A)$, and their intersection is $\mathrm{D}_{\mathrm{f}}^{\mathrm{b}}(\operatorname{Mod} A)$.

Definition 4.1 [Ye92, YZ99]. Let $A$ be a left noetherian $\mathbb{k}$-algebra and $B$ a right noetherian $\mathbb{k}$-algebra. A complex $R \in \mathrm{D}^{\mathrm{b}}\left(\operatorname{Mod}\left(A \otimes B^{\mathrm{op}}\right)\right)$ is called a dualizing complex over $(A, B)$ if it satisfies the following three conditions:

(i) $R$ has finite injective dimension over $A$ and over $B^{\text {op }}$;

(ii) $R$ has finite cohomology modules over $A$ and over $B^{\text {op }}$;

(iii) the canonical morphisms $B \rightarrow \operatorname{RHom}_{A}(R, R)$ in $\mathrm{D}\left(\operatorname{Mod} B^{\mathrm{e}}\right)$, and $A \rightarrow \operatorname{RHom}_{B^{\text {op }}}(R, R)$ in $\mathrm{D}\left(\operatorname{Mod} A^{\mathrm{e}}\right)$, are both isomorphisms.

In the case $A=B$, we say $R$ is a dualizing complex over $A$.

Whenever we refer to a dualizing complex over $(A, B)$, we tacitly assume that $A$ is left noetherian and $B$ is right noetherian.

Remark 4.2. There are many non-isomorphic dualizing complexes over a given $\mathbb{k}$-algebra $A$. The isomorphism classes of dualizing complexes are parameterized by the derived Picard group $\operatorname{DPic}(A)$, whose elements are the isomorphism classes of two-sided tilting complexes. See [Ye99] and [MY01].

We now give two easy examples.

Example 4.3. Suppose $A$ is a Gorenstein noetherian ring, namely the bimodule $R:=A$ has finite injective dimension as left and right module. Then $R$ is a dualizing complex over $A$. 


\section{A. YeKutieli And J. J. Zhang}

Example 4.4. If $A$ is a finite $\mathbb{k}$-algebra, then the bimodule $A^{*}:=\operatorname{Hom}_{\mathbb{k}}(A, \mathbb{k})$ is a dualizing complex over $A$. In fact it is a rigid dualizing complex (see Definition 4.10).

Definition 4.5. Let $R$ be a dualizing complex over $(A, B)$. The duality functors induced by $R$ are the contravariant functors

$$
\mathrm{D}:=\mathrm{RHom}_{A}(-, R): \mathrm{D}(\operatorname{Mod} A) \rightarrow \mathrm{D}\left(\operatorname{Mod} B^{\mathrm{op}}\right)
$$

and

$$
\mathrm{D}^{\mathrm{op}}:=\mathrm{RHom}_{B^{\mathrm{op}}}(-, R): \mathrm{D}\left(\operatorname{Mod} B^{\mathrm{op}}\right) \rightarrow \mathrm{D}(\operatorname{Mod} A) .
$$

By [YZ99, Proposition 1.3] the functors $\mathrm{D}$ and $\mathrm{D}^{\mathrm{op}}$ are a duality (i.e. an anti-equivalence) of triangulated categories between $\mathrm{D}_{\mathrm{f}}(\operatorname{Mod} A)$ and $\mathrm{D}_{\mathrm{f}}\left(\operatorname{Mod} B^{\mathrm{op}}\right)$, restricting to a duality between $\mathrm{D}_{\mathrm{f}}^{\mathrm{b}}(\operatorname{Mod} A)$ and $\mathrm{D}_{\mathrm{f}}^{\mathrm{b}}\left(\operatorname{Mod} B^{\mathrm{op}}\right)$.

Definition 4.6 [Ye96, YZ99]. Let $R$ be a dualizing complex over $(A, B)$. We say that $R$ has the Auslander property, or that $R$ is an Auslander dualizing complex, if the conditions below hold.

(i) For every finite $A$-module $M$, every integers $p>q$, and every $B^{\text {op }}$-submodule $N \subset \operatorname{Ext}_{A}^{p}(M, R)$, one has $\operatorname{Ext}_{B^{\mathrm{op}}}^{q}(N, R)=0$.

(ii) The same holds after exchanging $A$ and $B^{\text {op }}$.

Rings with Auslander dualizing complexes can be viewed as a generalization of Auslander regular rings (cf. [Bj89, Le92]).

Example 4.7. If $A$ is either the $n$th Weyl algebra or the universal enveloping algebra of a finitedimensional Lie algebra, then $A$ is Auslander regular, and the bimodule $R:=A$ is an Auslander dualizing complex.

Definition 4.8. An exact dimension function on $\operatorname{Mod} A$ is a function

$$
\operatorname{dim}: \operatorname{Mod} A \rightarrow\{-\infty\} \cup \mathbb{R} \cup\{\text { infinite ordinals }\},
$$

satisfying the following axioms:

(i) $\operatorname{dim} 0=-\infty$;

(ii) for every short exact sequence $0 \rightarrow M^{\prime} \rightarrow M \rightarrow M^{\prime \prime} \rightarrow 0$ one has $\operatorname{dim} M=\max \left\{\operatorname{dim} M^{\prime}\right.$, $\left.\operatorname{dim} M^{\prime \prime}\right\}$;

(iii) if $M=\bigcup_{\alpha} M_{\alpha}$ then $\operatorname{dim} M=\sup \left\{\operatorname{dim} M_{\alpha}\right\}$.

The basic examples of dimension functions are the Gelfand-Kirillov dimension, denoted by GKdim, and the Krull dimension, denoted by Kdim. See [MR87, Section 6.8.4]. We now give another dimension function.

Definition 4.9 [Ye96, YZ99]. Let $R$ be an Auslander dualizing complex over $(A, B)$. Given a finite $A$-module $M$ the canonical dimension of $M$ with respect to $R$ is

$$
\operatorname{Cdim}_{R ; A} M:=-\inf \left\{q \mid \operatorname{Ext}_{A}^{q}(M, R) \neq 0\right\} \in \mathbb{Z} \cup\{-\infty\} .
$$

For any $A$-module $M$ we define

$$
\operatorname{Cdim}_{R ; A} M:=\sup \left\{\operatorname{dim} M^{\prime} \mid M^{\prime} \subset M \text { is finite }\right\} .
$$

Likewise, we define $\operatorname{Cdim}_{R ; B^{\text {op }}} N$ for a $B^{\text {op }}$-module $N$.

Often we abbreviate $\operatorname{Cdim}_{R ; A}$ by dropping subscripts when no confusion can arise. According to [YZ99, Theorem 2.1], Cdim is an exact dimension function on $\operatorname{Mod} A$ and $\operatorname{Mod} B^{\text {op }}$. 


\section{DifFERENTIAL ALGEBRAS}

The following concept is due to Van den Bergh. Let $R$ be a dualizing complex over $A$. Since $R$ is a complex of $A^{\mathrm{e}}$-modules, the complex $R \otimes R$ consists of modules over $A^{\mathrm{e}} \otimes A^{\mathrm{e}} \cong\left(A^{\mathrm{e}}\right)^{\mathrm{e}}$. In the definition below, $\mathrm{RHom}_{A^{\mathrm{e}}}(A, R \otimes R)$ is computed using the 'outside' $A^{\mathrm{e}}$-module structure of $R \otimes R$, and the resulting complex retains the 'inside' $A^{\mathrm{e}}$-module structure.

Definition 4.10 [vdB97, Definition 8.1]. Let $R$ be a dualizing complex over $A$. If there is an isomorphism

$$
\rho: R \rightarrow \mathrm{RHom}_{A^{\mathrm{e}}}(A, R \otimes R)
$$

in $\mathrm{D}\left(\operatorname{Mod} A^{\mathrm{e}}\right)$, then we call $(R, \rho)$, or just $R$, a rigid dualizing complex. The isomorphism $\rho$ is called a rigidifying isomorphism.

A rigid dualizing complex, if it exists, is unique up to isomorphism, by [vdB97, Proposition 8.2].

A ring homomorphism $A \rightarrow B$ is said to be finite if $B$ is a finite $A$-module on both sides.

Definition 4.11 [YZ99, Definition 3.7]. Let $A \rightarrow B$ be a finite homomorphism of $\mathbb{k}$-algebras. Assume the rigid dualizing complexes $\left(R_{A}, \rho_{A}\right)$ and $\left(R_{B}, \rho_{B}\right)$ exist. Let $\operatorname{Tr}_{B / A}: R_{B} \rightarrow R_{A}$ be a morphism in $\mathrm{D}\left(\operatorname{Mod} A^{\mathrm{e}}\right)$. We say that $\operatorname{Tr}_{B / A}$ is a rigid trace if it satisfies the following two conditions.

(i) $\operatorname{Tr}_{B / A}$ induces isomorphisms

$$
R_{B} \cong \mathrm{RHom}_{A}\left(B, R_{A}\right) \cong \mathrm{RHom}_{A^{\text {op }}}\left(B, R_{A}\right)
$$

in $\mathrm{D}\left(\operatorname{Mod} A^{\mathrm{e}}\right)$.

(ii) The diagram

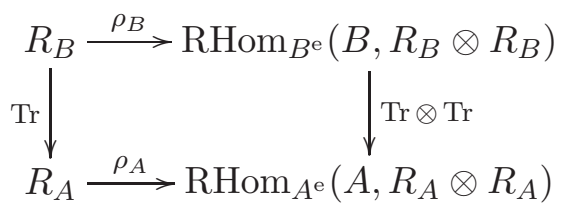

in $\mathrm{D}\left(\operatorname{Mod} A^{\mathrm{e}}\right)$ is commutative.

Often we say that $\operatorname{Tr}_{B / A}:\left(R_{B}, \rho_{B}\right) \rightarrow\left(R_{A}, \rho_{A}\right)$ is a rigid trace morphism.

By [YZ99, Theorem 3.2], a rigid trace $\operatorname{Tr}_{B / A}$ is unique (if it exists). In particular taking the identity map $A \rightarrow A$ and any two rigid dualizing complexes $(R, \rho)$ and $\left(R^{\prime}, \rho^{\prime}\right)$ over $A$, it follows there is a unique isomorphism $R \stackrel{\simeq}{\longrightarrow} R^{\prime}$ that is a rigid trace; see [YZ99, Corollary 3.4]. Given another finite homomorphism $B \rightarrow C$ such that the rigid dualizing complex $\left(R_{C}, \rho_{C}\right)$ and the rigid trace $\operatorname{Tr}_{C / B}$ exist, the composition $\operatorname{Tr}_{C / A}:=\operatorname{Tr}_{C / B} \circ \operatorname{Tr}_{B / A}$ is a rigid trace.

Finally we mention that by [YZ99, Corollary 3.6] the cohomology bimodules $\mathrm{H}^{i} R_{A}$ of the rigid dualizing complex are central $\mathrm{Z}(A)$-bimodules, where $\mathrm{Z}(A)$ is the center of $A$.

Example 4.12. Suppose that $A$ is a finitely generated commutative $\mathbb{k}$-algebra. Choose a finite homomorphism $\mathbb{k}[\boldsymbol{t}] \rightarrow A$ where $\mathbb{k}[\boldsymbol{t}]=\mathbb{k}\left[t_{1}, \ldots, t_{n}\right]$ is the polynomial algebra. Define $R_{A}:=$ $\operatorname{RHom}_{\mathbb{k}[t]}\left(A, \Omega_{\mathbb{R}[t] / k}^{n}[n]\right)$, and consider this as an object of $\mathrm{D}^{\mathrm{b}}\left(\operatorname{Mod} A^{\mathrm{e}}\right)$. By [Ye99, Proposition 5.7], the complex $R_{A}$ is an Auslander rigid dualizing complex, and in fact it is equipped with a canonical rigidifying isomorphism $\rho_{A}$.

\section{Quasi-coherent ringed schemes and localization}

In order to study geometric properties of dualizing complexes, it is convenient to use the language of schemes and quasi-coherent sheaves. See [YZ02, Theorems 0.1 and 0.2]. 


\section{A. YeKutieli And J. J. Zhang}

Let $(X, \mathcal{A})$ be a ringed space over $\mathbb{k}$. Thus $X$ is a topological space and $\mathcal{A}$ is a sheaf of (possibly noncommutative) $\mathbb{k}$-algebras on $X$. By an $\mathcal{A}$-bimodule we mean a sheaf $\mathcal{M}$ of $\mathbb{k}$-modules on $X$ together with a left $\mathcal{A}$-module structure and a right $\mathcal{A}$-module structure that commute with each other. In other words, $\mathcal{M}$ is a module over the sheaf of rings $\mathcal{A} \otimes_{\mathbb{k}_{X}} \mathcal{A}^{\text {op }}$, where $\mathbb{k}_{X}$ is the constant sheaf $\mathbb{k}$ on $X$. An $\mathcal{A}$-ring is a sheaf $\mathcal{B}$ of rings on $X$ together with a ring homomorphism $\mathcal{A} \rightarrow \mathcal{B}$. Note that $\mathcal{B}$ is an $\mathcal{A}$-bimodule.

Definition 5.1. Let $X$ be $\mathbb{k}$-scheme. An $\mathcal{O}_{X}$-ring $\mathcal{A}$ is called a quasi-coherent $\mathcal{O}_{X}$-ring if $\mathcal{A}$ is a quasi-coherent $\mathcal{O}_{X}$-module on both sides. The pair $(X, \mathcal{A})$ is then called a quasi-coherent ringed scheme.

Let $(X, \mathcal{A})$ be a quasi-coherent ringed scheme. An $\mathcal{A}$-module $\mathcal{M}$ is called quasi-coherent if locally, on every sufficiently small open set $U$, it has a free resolution

$$
\left.\left.\left.\mathcal{A}\right|_{U} ^{(J)} \rightarrow \mathcal{A}\right|_{U} ^{(I)} \rightarrow \mathcal{M}\right|_{U} \rightarrow 0
$$

cf. [EG71]. Equivalently, $\mathcal{M}$ is quasi-coherent as an $\mathcal{O}_{X}$-module. We denote the category of quasicoherent $\mathcal{A}$-modules by $\mathrm{Q} C o h \mathcal{A}$.

Proposition 5.2. Let $(X, \mathcal{A})$ be a quasi-coherent ringed scheme, let $U \subset X$ be an affine open set and $A:=\Gamma(U, \mathcal{A})$. The functor $\Gamma(U,-)$ is an equivalence of categories $\left.Q \operatorname{Coh} \mathcal{A}\right|_{U} \rightarrow \operatorname{Mod} A$.

Proof. This is a slight generalization of [EG71, Corollary 1.4.2 and Theorem 1.5.1]. See also [Ha77, Corollary II.5.5].

Given an $A$-module $M$, we usually denote the corresponding quasi-coherent $\left.\mathcal{A}\right|_{U}$-module by $\left.\mathcal{A}\right|_{U} \otimes_{A} M$.

The following definition is due to Silver [Si67, p. 47].

Definition 5.3. Let $A$ be a ring. An $A$-ring $A^{\prime}$ is called a localization of $A$ if $A^{\prime}$ is a flat $A$-module on both sides, and if the multiplication map $A^{\prime} \otimes_{A} A^{\prime} \rightarrow A^{\prime}$ is bijective.

Example 5.4. Let $A$ be a ring and $S \subset A$ a (left and right) denominator set. The ring of fractions $A_{S}$ of $A$ with respect to $S$ is the prototypical example of a localization of $A$. For reference we call such a localization an Ore localization.

We remind the reader that a denominator set $S$ is a multiplicatively closed subset of $A$ satisfying the left and right Ore conditions and the left and right torsion conditions (see [MR87, § 2.1]). The left Ore condition is that for all $a \in A$ and $s \in S$ there exist $a^{\prime} \in A$ and $s^{\prime} \in S$ such that $a s^{\prime}=a^{\prime} s$. The left torsion condition is

$$
\{a \in A \mid a s=0 \text { for some } s \in S\} \subset\{a \in A \mid s a=0 \text { for some } s \in S\} .
$$

The right Ore and torsion conditions for $A$ are the respective left conditions for $A^{\text {op }}$.

Not all localizations are Ore, as we see in Example 5.7.

Below we give a list of some nice descent properties enjoyed by localization, that are proved in $[\mathrm{Si} 67, \S 1]$.

Lemma 5.5. Let $A$ be a ring and let $A^{\prime}$ be a localization of $A$.

(1) For any $A^{\prime}$-module $M^{\prime}$ the multiplication $A^{\prime} \otimes_{A} M^{\prime} \rightarrow M^{\prime}$ is bijective.

(2) Let $M^{\prime}$ be an $A^{\prime}$-module and $M \subset M^{\prime}$ an $A$-submodule. Then the multiplication $A^{\prime} \otimes_{A} M \rightarrow M^{\prime}$ is injective.

(3) Let $M$ be an $A$-module and $\phi: M \rightarrow A^{\prime} \otimes_{A} M$ the homomorphism $\phi(m):=1 \otimes m$. Then for any $A^{\prime}$-submodule $N^{\prime} \subset A^{\prime} \otimes_{A} M$ the multiplication $A^{\prime} \otimes_{A} \phi^{-1}\left(N^{\prime}\right) \rightarrow N^{\prime}$ is bijective. 


\section{DifFERENTIAL ALGEBRAS}

(4) In the situation of part (3), the $A$-submodule $\phi(M) \subset A^{\prime} \otimes_{A} M$ is essential.

(5) Localization of a left noetherian ring is left noetherian.

Proposition 5.6. Let $(X, \mathcal{A})$ be a quasi-coherent ringed scheme, and let $V \subset U$ be affine open sets in $X$. Define $C:=\Gamma\left(U, \mathcal{O}_{X}\right), C^{\prime}:=\Gamma\left(V, \mathcal{O}_{X}\right), A:=\Gamma(U, \mathcal{A})$ and $A^{\prime}:=\Gamma(V, \mathcal{A})$.

(1) The multiplication maps $C^{\prime} \otimes_{C} A \rightarrow A^{\prime}$ and $A \otimes_{C} C^{\prime} \rightarrow A^{\prime}$ are bijective.

(2) $A \rightarrow A^{\prime}$ is a localization.

(3) Let $\mathcal{M}$ be a quasi-coherent $\mathcal{A}$-module. Then the multiplication map

$$
A^{\prime} \otimes_{A} \Gamma(U, \mathcal{M}) \rightarrow \Gamma(V, \mathcal{M})
$$

is bijective.

Proof. Define $C:=\Gamma\left(U, \mathcal{O}_{X}\right)$ and $C^{\prime}:=\Gamma\left(V, \mathcal{O}_{X}\right)$. We first show that $C^{\prime}$ is a localization of $C$, namely that $\phi: C \rightarrow C^{\prime}$ is flat and $\psi: C^{\prime} \otimes_{C} C^{\prime} \rightarrow C^{\prime}$ is bijective. This can be checked locally on $V$. Choose an affine open covering $V=\bigcup_{i} V_{i}$ with $V_{i}=\operatorname{Spec} C_{s_{i}}$ for suitable elements $s_{i} \in C$. We note that $C_{s_{i}} \cong C_{s_{i}}^{\prime} \cong \Gamma\left(V_{i}, \mathcal{O}_{X}\right)$ for all $i$. Hence, restricting $\phi$ and $\psi$ to $V_{i}$, namely applying $C_{s_{i}} \otimes_{C}-$ to them, we obtain bijections.

Let $\mathcal{M}$ be any quasi-coherent $\mathcal{A}$-module. By [Ha77, Proposition 5.1], multiplication

$$
C^{\prime} \otimes_{C} \Gamma(U, \mathcal{M}) \rightarrow \Gamma(V, \mathcal{M})
$$

is bijective.

Because $\mathcal{A}$ is a quasi-coherent left and right $\mathcal{O}_{X}$-module, the previous formula implies that $C^{\prime} \otimes_{C} A \rightarrow A^{\prime}$ and $A \otimes_{C} C^{\prime} \rightarrow A^{\prime}$ are both bijective. In addition, since we now know that

$$
A^{\prime} \otimes_{A} \Gamma(U, \mathcal{M}) \cong C^{\prime} \otimes_{C} \Gamma(U, \mathcal{M}),
$$

we may conclude that

$$
A^{\prime} \otimes_{A} \Gamma(U, \mathcal{M}) \rightarrow \Gamma(V, \mathcal{M})
$$

is bijective.

Finally we have a sequence of isomorphisms that are all compatible with the multiplication homomorphisms into $A^{\prime}$ :

$$
\begin{aligned}
A^{\prime} \otimes_{A} A^{\prime} & \cong A^{\prime} \otimes_{A}\left(A \otimes_{C} C^{\prime}\right) \cong A^{\prime} \otimes_{C} C^{\prime} \\
& \cong\left(A \otimes_{C} C^{\prime}\right) \otimes_{C} C^{\prime} \cong A \otimes_{C}\left(C^{\prime} \otimes_{C} C^{\prime}\right) \\
& \cong A \otimes_{C} C^{\prime} \cong A^{\prime} .
\end{aligned}
$$

Example 5.7. Let $X$ be an elliptic curve over $\mathbb{C}$ and $O \in X$ the zero element for the group structure. Let $P \in X$ be any nontorsion point. Define $U:=X-\{O\}$ and $V:=X-\{O, P\}$, which are affine open sets, and $C:=\Gamma\left(U, \mathcal{O}_{X}\right), C^{\prime}:=\Gamma\left(V, \mathcal{O}_{X}\right)$. By the previous proposition $C \rightarrow C^{\prime}$ is a localization. We claim this is not an Ore localization. If it were an Ore localization, then there would be some noninvertible nonzero function $s \in C$ that becomes invertible in $C^{\prime}$. Hence, the divisor of $s$ on $X$ would be $(s)=n(O-P)$ for some positive integer $n$. In the group structure this would mean that $P$ is a torsion point, and this is a contradiction.

Definition 5.8. Let $A$ be a ring, let $M$ be an $A$-bimodule and let $A^{\prime}$ be a localization of $A$. If the canonical homomorphisms

$$
A^{\prime} \otimes_{A} M \rightarrow A^{\prime} \otimes_{A} M \otimes_{A} A^{\prime}
$$

and

$$
M \otimes_{A} A^{\prime} \rightarrow A^{\prime} \otimes_{A} M \otimes_{A} A^{\prime}
$$

are bijective, then $M$ is said to be evenly localizable to $A^{\prime}$. 


\section{A. YeKutieli And J. J. Zhang}

Trivially the bimodule $M:=A$ is evenly localizable to $A^{\prime}$. The next lemma is also easy and we omit its proof.

Lemma 5.9. Let $M$ be an A-bimodule. Suppose that $A^{\prime}$ is a localization of $A$.

(1) If $A$ is commutative, $M$ is a central $A$-bimodule and $A^{\prime}$ is an $A$-algebra, then $M$ is evenly localizable to $A^{\prime}$.

(2) If there is a short exact sequence of $A$-bimodules $0 \rightarrow L \rightarrow M \rightarrow N \rightarrow 0$ with $L$ and $N$ evenly localizable to $A^{\prime}$, then $M$ is also evenly localizable to $A^{\prime}$.

(3) Suppose that $M \cong \lim _{i \rightarrow} M_{i}$ for some directed system of $A$-bimodules $\left\{M_{i}\right\}$. If each $M_{i}$ is evenly localizable to $A^{\prime}$, then so is $M$.

Proposition 5.10. Let $C$ be a commutative ring and let $M$ be a $C$-bimodule. Define $U:=\operatorname{Spec} C$. The following conditions are equivalent.

(i) For any multiplicatively closed subset $S \subset C$, with localization $C_{S}, M$ is evenly localizable to $C_{S}$.

(ii) $M$ is evenly localizable to $C^{\prime}:=\Gamma\left(V, \mathcal{O}_{U}\right)$ for every affine open set $V \subset U$.

(iii) There is a sheaf of $\mathcal{O}_{U}$-bimodules $\mathcal{M}$, quasi-coherent on both sides, with $M \cong \Gamma(U, \mathcal{M})$. Such $\mathcal{M}$ is unique up to a unique isomorphism.

Proof. (i) $\Rightarrow$ (ii) Let us write

$$
\phi: C^{\prime} \otimes_{C} M \rightarrow C^{\prime} \otimes_{C} M \otimes_{C} C^{\prime} .
$$

As in the proof of Proposition 5.6 we choose an affine open covering $V=\bigcup_{i} V_{i}$ with $V_{i}=\operatorname{Spec} C_{s_{i}}$ and $C_{s_{i}}^{\prime} \cong C_{s_{i}}$. It suffices to show that the homomorphism $\phi_{i}$ obtained by applying $C_{s_{i}}^{\prime} \otimes_{C^{\prime}}-$ to $\phi$ (localizing on the left) is bijective for all $i$. Using the hypothesis (i) with $S:=\left\{s_{i}^{l}\right\}_{l \in \mathbb{N}}$ and the fact that $C_{s_{i}} \rightarrow C_{s_{i}}^{\prime}$ is bijective we get

$$
C_{s_{i}}^{\prime} \otimes_{C^{\prime}}\left(C^{\prime} \otimes_{C} M\right) \cong C_{s_{i}} \otimes_{C} M \otimes_{C} C_{s_{i}}
$$

and

$$
\begin{aligned}
C_{s_{i}}^{\prime} \otimes_{C^{\prime}}\left(C^{\prime} \otimes_{C} M \otimes_{C} C^{\prime}\right) & \cong C_{s_{i}} \otimes_{C} M \otimes_{C} C_{s_{i}} \otimes_{C} C^{\prime} \\
& \cong C_{s_{i}} \otimes_{C} M \otimes_{C} C_{s_{i}} .
\end{aligned}
$$

So $\phi_{i}$ is bijective.

Similarly, one shows that

$$
M \otimes_{C} C^{\prime} \rightarrow C^{\prime} \otimes_{C} M \otimes_{C} C^{\prime}
$$

is bijective.

(ii) $\Rightarrow$ (i) For any element $s \in S$ let $V:=\operatorname{Spec} C_{s} \subset U$. By assumption,

$$
C_{s} \otimes_{C} M \cong C_{s} \otimes_{C} M \otimes_{C} C_{s} \cong M \otimes_{C} C_{s} .
$$

Taking direct limit over $s \in S$, we get

$$
C_{S} \otimes_{C} M \cong C_{S} \otimes_{C} M \otimes_{C} C_{S} \cong M \otimes_{C} C_{S} .
$$

(ii) $\Rightarrow$ (iii) Let $\mathcal{M}:=\mathcal{O}_{U} \otimes_{C} M$ be the sheafification of the (left) $C$-module $M$ to $U$. By definition, $\mathcal{M}$ is a quasi-coherent left $\mathcal{O}_{U}$-module.

Given an affine open set $V \subset U$ write $C^{\prime}:=\Gamma\left(V, \mathcal{O}_{U}\right)$. By Proposition 5.6 the multiplication map $C^{\prime} \otimes_{C} M \rightarrow \Gamma(V, \mathcal{M})$ is a bijection. Therefore, $\Gamma(V, \mathcal{M}) \cong C^{\prime} \otimes_{C} M \otimes_{C} C^{\prime}$. Since $M$ is evenly localizable to $C^{\prime}$, it follows that $M \otimes_{C} C^{\prime} \rightarrow \Gamma(V, \mathcal{M})$ is also bijective. We conclude that $\mathcal{M}$ is also a quasi-coherent right $\mathcal{O}_{U}$-module. 


\section{Differential ALGEBRAS}

Regarding the uniqueness, suppose that $\mathcal{N}$ is another $\mathcal{O}_{U}$-bimodule quasi-coherent on both sides such that $\Gamma(U, \mathcal{N}) \cong M$ as bimodules. For any affine open set $V$ as above, we get an isomorphism of $C^{\prime}$-bimodules

$$
\Gamma(V, \mathcal{M}) \cong C^{\prime} \otimes_{C} M \otimes_{C} C^{\prime} \cong \Gamma(V, \mathcal{N})
$$

which is functorial in $V$. Therefore, $\mathcal{M} \cong \mathcal{N}$ as $\mathcal{O}_{U}$-bimodules.

(iii) $\Rightarrow$ (ii) Since $\mathcal{M}$ is quasi-coherent on both sides, for any affine open set $V=\operatorname{Spec} C^{\prime}$ we have

$$
\Gamma(V, \mathcal{M})=C^{\prime} \otimes_{C} M=M \otimes_{C} C^{\prime},
$$

so $M$ is evenly localizable to $C^{\prime}$.

The relation between even localization and Ore localization of a ring is explained in the next theorem.

Theorem 5.11. Let $C$ be a commutative ring, let $A$ be a $C$-ring and $S \subset C$ a multiplicatively closed subset. Denote by $C_{S}$ the ring of fractions of $C$ with respect to $S$. Then the following two conditions are equivalent.

(i) The image $\bar{S}$ of $S$ in $A$ is a denominator set, with ring of fractions $A_{\bar{S}}$.

(ii) The $C$-bimodule $A$ is evenly localizable to $C_{S}$.

When these conditions hold, the multiplication map

$$
C_{S} \otimes_{C} A \otimes_{C} C_{S} \rightarrow A_{\bar{S}}
$$

is bijective.

Proof. (i) $\Rightarrow$ (ii) Since $A_{\bar{S}}$ is the left ring of fractions of $A$ with respect to $\bar{S}$ (see [MR87, $\left.\S 2.1 .3\right]$ ), it follows that the homomorphism $C_{S} \otimes_{C} A \rightarrow A_{\bar{S}}$ is bijective. On the other hand, since $A_{\bar{S}}$ is also the right ring of fractions, $A \otimes_{C} C_{S} \rightarrow A_{\bar{S}}$ is bijective.

$$
\text { (ii) } \Rightarrow \text { (i) Write }
$$

$$
Q:=C_{S} \otimes_{C} A \otimes_{C} C_{S}
$$

and

$$
\phi: A \rightarrow Q, \quad \phi(a):=1 \otimes a \otimes 1 .
$$

The assumption that $A$ is evenly localizable to $C_{S}$ implies that

$$
\begin{aligned}
\operatorname{Ker}(\phi) & =\{a \in A \mid a s=0 \text { for some } s \in S\} \\
& =\{a \in A \mid s a=0 \text { for some } s \in S\},
\end{aligned}
$$

verifying the torsion conditions.

The even localization assumption also implies that given $a_{1} \in A$ and $s_{1} \in S$ there are $a_{2} \in A$ and $s_{2} \in S$ such that

$$
s_{1}^{-1} \otimes a_{1} \otimes 1=1 \otimes a_{2} \otimes s_{2}^{-1} \in Q .
$$

Multiplying this equation by $s_{1}$ on the left and by $s_{2}$ on the right we obtain $1 \otimes a_{1} s_{2} \otimes 1=$ $1 \otimes s_{1} a_{2} \otimes 1$. Therefore, $\phi\left(s_{1} a_{2}-a_{1} s_{2}\right)=0$. Since $Q \cong A \otimes_{C} C_{S}$ there exists some $s_{3} \in S$ such that $\left(s_{1} a_{2}-a_{1} s_{2}\right) s_{3}=0$ in $A$, i.e.

$$
s_{1}\left(a_{2} s_{3}\right)=a_{1}\left(s_{2} s_{3}\right) \in A .
$$

We have verified the right Ore condition. The left Ore condition is verified the same way.

Remark 5.12. The theorem applies to any ring $A$ and any commutative multiplicatively closed subset $S \subset A$, since we can take $C:=\mathbb{Z}[S] \subset A$.

We will need a geometric interpretation of Theorem 5.11. 


\section{A. YeKutieli And J. J. Zhang}

Corollary 5.13. Let $C$ be a commutative ring, let $U:=\operatorname{Spec} C$ and let $A$ be a $C$-ring. The following conditions are equivalent:

(i) for every multiplicatively closed set $S \subset C$ the $C$-bimodule $A$ is evenly localizable to $C_{S}$;

(ii) for every multiplicatively closed set $S \subset C$ its image $\bar{S} \subset A$ is a denominator set;

(iii) there is a quasi-coherent $\mathcal{O}_{U}$-ring $\mathcal{A}$ such that $\Gamma(U, \mathcal{A}) \cong A$ as $C$-rings.

When these conditions hold the quasi-coherent $\mathcal{O}_{U}$-ring $\mathcal{A}$ is unique up to a unique isomorphism.

Proof. (i) and (ii) are equivalent by Theorem 5.11. The implication (iii) $\Rightarrow$ (i) is a special case of Proposition 5.10. It remains to show that (i) $\Rightarrow$ (iii).

By Proposition 5.10 there is an $\mathcal{O}_{U}$-bimodule $\mathcal{A}$, quasi-coherent on both sides, such that $A \cong \Gamma(U, \mathcal{A})$ as $C$-bimodules. The bimodule $\mathcal{A}$ is unique up to a unique isomorphism. Next, by Theorem 5.11, for any $s \in C$, letting $S:=\left\{s^{i}\right\}_{i \in \mathbb{N}}$, the image $\bar{S} \subset A$ is a denominator set. Therefore, on $V:=\operatorname{Spec} C_{s}$ we have canonical isomorphisms

$$
\Gamma(V, \mathcal{A}) \cong C_{s} \otimes_{C} A \otimes_{C} C_{s} \cong A_{\bar{S}}
$$

where $A_{\bar{S}}$ is the ring of fractions of $A$ with respect to $\bar{S}$. Hence, $\mathcal{A}$ has a unique structure of quasi-coherent $\mathcal{O}_{X}$-ring.

Here is an (somewhat artificial) example of a $C$-ring $A$ satisfying the conditions of Corollary 5.13, but the $C^{\mathrm{e}}$-ring $A^{\mathrm{e}}$ fails to satisfy them.

Example 5.14. Let $C:=\mathbb{Q}[t]$ with $t$ a variable, and let $U:=\operatorname{Spec} C$. Take $A:=\mathbb{Q}(t)[a ; \sigma]$, an Ore extension of the field $\mathbb{Q}(t)$, where $\sigma$ is the automorphism $\sigma(t)=-t$. Since every nonzero element $s \in C$ is invertible in $A$, the $C$-ring $A$ is evenly localizable to $C_{S}$ for any multiplicatively closed subset $S \subset C$. Hence, there is a quasi-coherent ringed scheme $(U, \mathcal{A})$ with $\Gamma(U, \mathcal{A}) \cong A$ as $C$-rings. (In fact, $\mathcal{A}$ is a constant sheaf on $U$.) Likewise there is a quasi-coherent ringed scheme $\left(U, \mathcal{A}^{\text {op }}\right)$.

We claim that there does not exist a quasi-coherent ringed scheme $\left(U^{2}, \mathcal{A}^{\mathrm{e}}\right)$ such that $\Gamma\left(U^{2}, \mathcal{A}^{\mathrm{e}}\right) \cong$ $A^{\mathrm{e}}$ as $C^{\mathrm{e}}$-rings. By Corollary 5.13, it suffices to exhibit a multiplicatively closed subset $S \subset C^{\mathrm{e}}$ that is not a denominator set in $A^{\mathrm{e}}$. Consider the element $s:=t \otimes 1-1 \otimes t \in C^{\mathrm{e}}$ and the set $S:=\left\{s^{n}\right\}_{n \in \mathbb{N}}$. Let $\mu: A^{\mathrm{e}} \rightarrow A$ be the multiplication map $\mu\left(a_{1} \otimes a_{2}\right):=a_{1} a_{2}$, which is a homomorphism of (left) $A^{\mathrm{e}}$-modules, and denote by $I$ the left ideal $\operatorname{Ker}(\mu)$. Then $A^{\mathrm{e}} \cdot s \subset I$. On the other hand $s(a \otimes 1)=t a \otimes 1-a \otimes t$, so $\mu(s(a \otimes 1))=t a-a t=2 t a$, and by induction $\mu\left(s^{n}(a \otimes 1)\right)=(2 t)^{n} a \neq 0$ for all $n \geqslant 0$. We conclude that $s^{n}(a \otimes 1) \notin A^{\mathrm{e}} \cdot s$, so $S$ fails to satisfy the left Ore condition.

Example 5.15. The quasi-coherent ringed scheme $(U, \mathcal{A})$ of the previous example also has the following peculiarity: the $C^{\mathrm{e}}$-module $A$ is not supported on the diagonal $\Delta(U) \subset U^{2}$. Indeed, for every $n \geqslant 0$ one has $s^{n} a=(2 t)^{n} a \neq 0$.

Definition 5.16. Let $C$ be a commutative $\mathbb{k}$-algebra and $M$ a $C$-bimodule. A differential $C$-filtration on $M$ is an exhaustive, bounded below filtration $F=\left\{F_{i} M\right\}$ where each $F_{i} M$ is a $C$-sub-bimodule, and $\operatorname{gr}^{F} M$ is a central $C$-bimodule. If $M$ admits some differential $C$-filtration then we call $M$ a differential $C$-bimodule.

Localization of a ring was defined in Definition 5.3, and even localization of a bimodule was introduced in Definition 5.8.

Proposition 5.17. Let $C$ be a commutative $\mathbb{k}$-algebra and let $M$ be a differential $C$-bimodule. If $C^{\prime}$ is a localization of $C$, then $M$ is evenly localizable to $C^{\prime}$.

Proof. If $M$ is a central $C$-bimodule, then according to Lemma 5.9(1) $M$ is evenly localizable to $C^{\prime}$. 


\section{DifFERENTIAL ALGEBRAS}

Now let $M$ be a $C$-bimodule equipped with a differential $C$-filtration $F$. Say $F_{i_{0}-1} M=0$. We prove by induction on $i \geqslant i_{0}$ that $F_{i} M$ is evenly localizable to $C^{\prime}$. First $F_{i_{0}} M$ is central, so the above applies to it. For any $i$ there is an exact sequence

$$
0 \rightarrow F_{i-1} M \rightarrow F_{i} M \rightarrow \operatorname{gr}_{i}^{F} M \rightarrow 0 .
$$

By the previous paragraph and by the induction hypothesis $F_{i-1} M$ and $\operatorname{gr}_{i}^{F} M$ are evenly localizable to $C^{\prime}$. The flatness of $C \rightarrow C^{\prime}$ extends this to $F_{i} M$, see Lemma 5.9(2). Finally we use Lemma 5.9(3).

Corollary 5.18. Let $C$ be a finitely generated commutative $\mathbb{k}$-algebra, $A$ a differential $C$-ring of finite type, $s \in C$ and $S:=\left\{s^{i}\right\}_{i \in \mathbb{N}}$. Then:

(1) the image $\bar{S}$ of $S$ in $A$ is a denominator set;

(2) let $C_{s}$ and $A_{s}$ be the Ore localizations $S$, then $C_{s}$ is a finitely generated $\mathbb{k}$-algebra and $A_{s}$ is a differential $C_{s}$-ring of finite type.

Proof. (1) Use Proposition 5.17 and Theorem 5.11.

(2) Suppose that $F=\left\{F_{i} A\right\}$ is a differential $C$-filtration of finite type. Then setting

$$
F_{i} A_{s}:=C_{s} \otimes_{C}\left(F_{i} A\right) \otimes_{C} C_{s} \subset A_{s}
$$

we obtain a filtration $F$ of $A_{s}$ such that $\operatorname{gr}^{F} A_{s} \cong C_{s} \otimes_{C} \operatorname{gr} F A$ as graded $C_{s^{-}}$algebras.

Remark 5.19. The ideas in [KL00, Theorem 4.9] can be used to show the following. In the setup of the previous corollary let $M$ be a finite $A$-module and $\bar{M}:=\operatorname{Im}\left(M \rightarrow A_{s} \otimes_{A} M\right)$. Then

$$
\operatorname{GKdim}_{A} \bar{M}=\operatorname{GKdim}_{A_{s}}\left(A_{s} \otimes_{A} M\right) .
$$

Corollary 5.20. Let $C$ be a commutative $\mathbb{k}$-algebra, let $U:=\operatorname{Spec} C$ and let $A$ be a differential $C$-ring.

(1) There is a quasi-coherent $\mathcal{O}_{U}$-ring $\mathcal{A}$, unique up to a unique isomorphism, such that $\Gamma(U, \mathcal{A}) \cong A$ as $C$-rings.

(2) For any multiplicatively closed set $S \subset C$ its image $\bar{S} \subset A$ is a denominator set.

(3) Given an affine open set $V \subset U$ let $C^{\prime}:=\Gamma\left(V, \mathcal{O}_{U}\right)$. Then $A^{\prime}:=C^{\prime} \otimes_{C} A \otimes_{C} C^{\prime}$ is a $\mathbb{k}$-algebra and $A \rightarrow A^{\prime}$ is a localization of rings. If $A$ is noetherian then so is $A^{\prime}$.

Proof. $A$ is a differential $C$-bimodule, so by Proposition $5.17 A$ is evenly localizable to $C_{S}$ for any multiplicatively closed set $S \subset C$. By Corollary 5.13 there is a quasi-coherent $\mathcal{O}_{U}$-ring $\mathcal{A}$, and by Proposition 5.6 we have $A^{\prime} \cong \Gamma(V, \mathcal{A})$. The assertion about noetherian rings follows from Lemma 5.5(5).

Proposition 5.21. Let $C$ be a commutative $\mathbb{k}$-algebra, let $U:=\operatorname{Spec} C$, let $M$ be a $C^{\mathrm{e}}$-module and let $\mathcal{M}:=\mathcal{O}_{U^{2}} \otimes_{C}$ e $M$, the quasi-coherent $\mathcal{O}_{U^{2}}$-module associated to $M$. Assume $C^{\mathrm{e}}$ is noetherian. Then the following conditions are equivalent:

(i) $M$ is a differential $C$-bimodule;

(ii) $\mathcal{M}$ is supported on the diagonal $\Delta(U) \subset U^{2}$.

Proof. (i) $\Rightarrow$ (ii) Denote by $I:=\operatorname{Ker}\left(C^{\mathrm{e}} \rightarrow C\right)$ and $\mathcal{I}:=\mathcal{O}_{U^{2}} \otimes_{C^{\mathrm{e}}} I$. So $\mathcal{I}$ is an ideal defining the diagonal $\Delta(U)$. Suppose $F=\left\{F_{i} M\right\}$ is a differential $C$-filtration of $M$, with $F_{i_{0}-1} M=0$. Then for all $i \geqslant i_{0}$ we have

$$
I^{i-i_{0}+1} \cdot F_{i} M=0 .
$$

It follows that the $\mathcal{O}_{U^{2}}$-module $F_{i} \mathcal{M}:=\mathcal{O}_{U^{2}} \otimes_{C^{\mathrm{e}}} F_{i} M$ is supported on $\Delta(U)$. However, $\mathcal{M}=\bigcup F_{i} \mathcal{M}$. 


\section{A. YeKutieli And J. J. Zhang}

(ii) $\Rightarrow$ (i) Let $\left\{\mathcal{M}_{\alpha}\right\}$ be the set of coherent $\mathcal{O}_{U^{2}}$-submodules of $\mathcal{M}$, so $\mathcal{M}=\bigcup \mathcal{M}_{\alpha}$. Now $\mathcal{M}_{\alpha}$ is a coherent $\mathcal{O}_{U^{2}}$-module supported on the diagonal $\Delta(U)$, so there is some integer $i_{\alpha} \geqslant 0$ such that $\mathcal{I}^{i_{\alpha}+1} \cdot \mathcal{M}_{\alpha}=0$. It follows that the $C^{\mathrm{e}}$-module $M_{\alpha}:=\Gamma\left(U^{2}, \mathcal{M}_{\alpha}\right)$ satisfies $I^{i_{\alpha}+1} \cdot M=0$. And $M=\bigcup M_{\alpha}$.

Define a filtration $F$ on $M$ by $F_{i} M:=\operatorname{Hom}_{C^{\mathrm{e}}}\left(C^{\mathrm{e}} / I^{i+1}, M\right)$ for $i \geqslant 0$, and $F_{-1} M:=0$. Then $M_{\alpha} \subset F_{i_{\alpha}} M$, and this implies that $M=\bigcup F_{i} M$. Finally $I \cdot F_{i} M \subset F_{i-1} M$, and hence $\operatorname{gr}_{i}^{F} M$ is a central $C$-bimodule.

\section{Localization of dualizing complexes}

In this section we study the behavior of rigid dualizing complexes over rings with respect to localization (cf. Definition 5.8).

Definition 6.1. Let $A \rightarrow A^{\prime}$ be a localization homomorphism between two noetherian $\mathbb{k}$-algebras. Suppose the rigid dualizing complexes $(R, \rho)$ and $\left(R^{\prime}, \rho^{\prime}\right)$ of $A$ and $A^{\prime}$, respectively, exist. A rigid localization morphism is a morphism

$$
\mathrm{q}_{A^{\prime} / A}: R \rightarrow R^{\prime}
$$

in $\mathrm{D}\left(\operatorname{Mod} A^{\mathrm{e}}\right)$ satisfying the conditions below.

(i) The morphisms $A^{\prime} \otimes_{A} R \rightarrow R^{\prime}$ and $R \otimes_{A} A^{\prime} \rightarrow R^{\prime}$ induced by $\mathrm{q}_{A^{\prime} / A}$ are isomorphisms.

(ii) The diagram

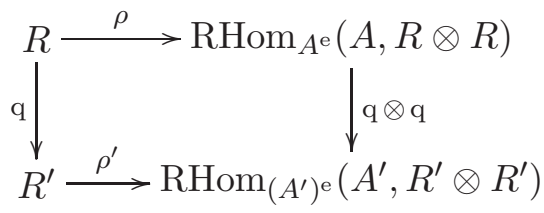

in $\mathrm{D}\left(\operatorname{Mod} A^{\mathrm{e}}\right)$ is commutative, where $\mathrm{q}:=\mathrm{q}_{A^{\prime} / A}$.

We sometimes express this by saying that $\mathrm{q}_{A^{\prime} / A}:(R, \rho) \rightarrow\left(R^{\prime}, \rho^{\prime}\right)$ is a rigid localization morphism.

We now give a generalization of [YZ03, Theorem 3.8].

Theorem 6.2. Let $A$ be a noetherian $\mathbb{k}$-algebra and let $A^{\prime}$ be a localization of $A$. Assume that $A$ has a dualizing complex $R$ such that the cohomology bimodules $\mathrm{H}^{i} R$ are evenly localizable to $A^{\prime}$. Then we have the following.

(1) The complex

$$
R^{\prime}:=A^{\prime} \otimes_{A} R \otimes_{A} A^{\prime}
$$

is a dualizing complex over $A^{\prime}$.

(2) If $R$ is an Auslander dualizing complex over $A$, then $R^{\prime}$ is an Auslander dualizing complex over $A^{\prime}$.

(3) Suppose $R$ is a rigid dualizing complex over $A$ with rigidifying isomorphism $\rho$, and $A^{\mathrm{e}}$ is noetherian. Then $R^{\prime}$ is a rigid dualizing complex over $A^{\prime}$. Furthermore $R^{\prime}$ has a unique rigidifying isomorphism $\rho^{\prime}$ such that the morphism $\mathrm{q}_{A^{\prime} / A}: R \rightarrow R^{\prime}$ defined by $r \mapsto 1 \otimes r \otimes 1$ is a rigid localization morphism.

(4) In the situation of part (3), the rigid localization morphism $\mathrm{q}_{A^{\prime} / A}:(R, \rho) \rightarrow\left(R^{\prime}, \rho^{\prime}\right)$ is unique.

Proof. (1) This follows essentially from the proof of [YZ99, Theorem 1.13]. There $A$ was commutative and $A^{\prime}$ was the localization of $A$ at some prime ideal, but the arguments are valid for an arbitrary localization $A^{\prime}$. 


\section{DifFERENTIAL ALGEBRAS}

(2) To check the Auslander property for $R^{\prime}$ let $M^{\prime}$ be any finite $A^{\prime}$-module. By Lemma 5.5(2) there is a finite $A$-module $M$ such that $M^{\prime} \cong A^{\prime} \otimes_{A} M$. For any $i$, [YZ03, Lemma 3.7(1)] implies that

$$
\operatorname{Ext}_{A^{\prime}}^{i}\left(M^{\prime}, R^{\prime}\right) \cong \operatorname{Ext}_{A}^{i}\left(M, R^{\prime}\right) \cong \operatorname{Ext}_{A}^{i}(M, R) \otimes_{A} A^{\prime}
$$

as $\left(A^{\prime}\right)^{\text {op }}$-modules. Given any $\left(A^{\prime}\right)^{\mathrm{op}}$-submodule $N^{\prime} \subset \operatorname{Ext}_{A^{\prime}}^{i}\left(M^{\prime}, R^{\prime}\right)$, Lemma 5.5(3) tells us that there is an $A^{\text {op }}$-submodule $N \subset \operatorname{Ext}_{A}^{i}(M, R)$ such that $N^{\prime} \cong N \otimes_{A} A^{\prime}$. For such $N$ we have

$$
\operatorname{Ext}_{\left(A^{\prime}\right)^{\text {op }}}^{j}\left(N^{\prime}, R^{\prime}\right) \cong A^{\prime} \otimes_{A} \operatorname{Ext}_{A^{\text {op }}}^{j}(N, R)
$$

which is 0 for all $j<i$. By symmetry we get the other half of the Auslander property for $R^{\prime}$.

(3) As in the proof of [YZ03, Theorem 3.8(2)] we have a canonical isomorphism

$$
A^{\prime} \otimes_{A} \operatorname{RHom}_{A^{\mathrm{e}}}(A, R \otimes R) \otimes_{A} A^{\prime} \stackrel{\simeq}{\longrightarrow} \operatorname{RHom}_{\left(A^{\prime}\right) \mathrm{e}}\left(A^{\prime}, R^{\prime} \otimes R^{\prime}\right)
$$

in $\mathrm{D}\left(\operatorname{Mod}\left(A^{\prime}\right)^{\mathrm{e}}\right)$. This defines a rigidifying isomorphism $\rho^{\prime}$ that respects $\mathrm{q}_{A^{\prime} / A}$ as depicted in the diagram in Definition 6.1. Given any other morphism

$$
\rho^{\prime \prime}: R^{\prime} \rightarrow \operatorname{RHom}_{\left(A^{\prime}\right)^{\mathrm{e}}}\left(A^{\prime}, R^{\prime} \otimes R^{\prime}\right)
$$

that renders the diagram commutative, applying the functor $A^{\prime} \otimes_{A}-\otimes_{A} A^{\prime}$ to the whole diagram we deduce that $\rho^{b}=\rho^{\prime}$.

(4) Write $\mathrm{q}_{1}:=\mathrm{q}_{A / A^{\prime}}$. Suppose $\mathrm{q}_{2}:(R, \rho) \rightarrow\left(R^{\prime}, \rho^{\prime}\right)$ is another rigid localization morphism. Consider the commutative diagrams

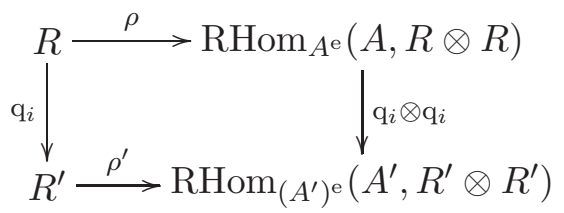

in $\mathrm{D}\left(\operatorname{Mod} A^{\mathrm{e}}\right)$. Applying the base change functor $\left(A^{\prime}\right)^{\mathrm{e}} \otimes_{A^{\mathrm{e}}}-$ to these diagrams we obtain diagrams

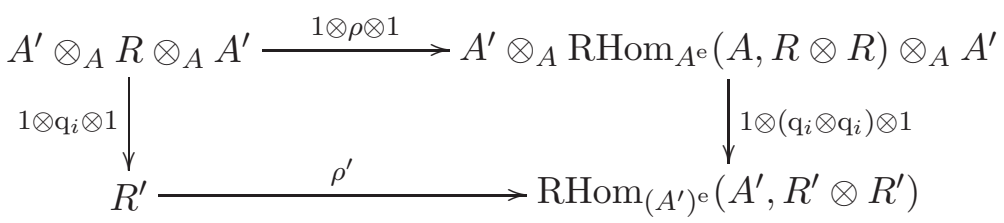

consisting of isomorphisms in $\mathrm{D}\left(\operatorname{Mod}\left(A^{\prime}\right)^{\mathrm{e}}\right)$; cf. [YZ03, Proof of Theorem 3.8(2)]. We obtain an isomorphism $\tau: R^{\prime} \rightarrow R^{\prime}$ such that

$$
1 \otimes \mathrm{q}_{2} \otimes 1=\tau \circ\left(1 \otimes \mathrm{q}_{1} \otimes 1\right): A^{\prime} \otimes_{A} R \otimes_{A} A^{\prime} \rightarrow R^{\prime} .
$$

However, then $\tau:\left(R^{\prime}, \rho^{\prime}\right) \rightarrow\left(R^{\prime}, \rho^{\prime}\right)$ is a rigid trace morphism. By [YZ03, Theorem 3.2], $\tau$ has to be the identity. This implies $1 \otimes \mathrm{q}_{2} \otimes 1=1 \otimes \mathrm{q}_{1} \otimes 1$ and therefore $\mathrm{q}_{2}=\mathrm{q}_{1}$.

The next proposition guarantees that under suitable assumptions the rigid trace localizes.

Proposition 6.3. Let

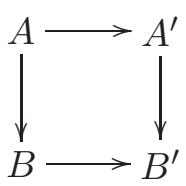

be a commutative diagram of $\mathbb{k}$-algebras, where the horizontal arrows are localizations, the vertical arrows are finite, and the multiplication maps $A^{\prime} \otimes_{A} B \rightarrow B^{\prime}$ and $B \otimes_{A} A^{\prime} \rightarrow B^{\prime}$ are bijective. Assume $A, A^{\prime}, A^{\mathrm{e}}, B, B^{\prime}$ and $B^{\mathrm{e}}$ are all noetherian. Also assume the rigid dualizing complexes $\left(R_{A}, \rho_{A}\right)$ 


\section{A. YeKutieli And J. J. Zhang}

and $\left(R_{B}, \rho_{B}\right)$ exist, and so does the rigid trace morphism $\operatorname{Tr}_{B / A}: R_{B} \rightarrow R_{A}$. By Theorem 6.2 the complexes $R_{A^{\prime}}:=A^{\prime} \otimes_{A} R_{A} \otimes_{A} A^{\prime}$ and $R_{B^{\prime}}:=B^{\prime} \otimes_{B} R_{B} \otimes_{B} B^{\prime}$ are rigid dualizing complexes over $A^{\prime}$ and $B^{\prime}$, respectively, with induced rigidifying isomorphisms $\rho_{A^{\prime}}$ and $\rho_{B^{\prime}}$. Then the morphism

$$
\operatorname{Tr}_{B^{\prime} / A^{\prime}}:=1 \otimes \operatorname{Tr}_{B / A} \otimes 1: R_{B^{\prime}} \rightarrow R_{A^{\prime}}
$$

is a rigid trace.

Proof. We begin by showing that the morphism $\psi^{\prime}: R_{B^{\prime}} \rightarrow \mathrm{RHom} A_{A^{\prime}}\left(B^{\prime}, R_{A^{\prime}}\right)$ induced by $\operatorname{Tr}_{B^{\prime} / A^{\prime}}$ is an isomorphism. Let us recall how $\psi^{\prime}$ is defined: one chooses a quasi-isomorphism $R_{A^{\prime}} \rightarrow I^{\prime}$ where $I^{\prime}$ is a bounded below complex of injective $\left(A^{\prime}\right)^{\mathrm{e}}$-modules. Then $\operatorname{Tr}_{B^{\prime}} / A^{\prime}$ is represented by an actual homomorphism of complexes $\tau^{\prime}: R_{B^{\prime}} \rightarrow I^{\prime}$. The formula for $\psi^{\prime}: R_{B^{\prime}} \rightarrow \operatorname{Hom}_{A^{\prime}}\left(B^{\prime}, I^{\prime}\right)$ is $\psi^{\prime}\left(\beta^{\prime}\right)\left(b^{\prime}\right)=\tau^{\prime}\left(b^{\prime} \beta^{\prime}\right)$ for $\beta^{\prime} \in R_{B^{\prime}}$ and $b^{\prime} \in B^{\prime}$.

Let $R_{A} \rightarrow I$ be a quasi-isomorphism where $I$ is a bounded below complex of injective $A^{\mathrm{e}}$-modules, and let $\tau: R_{B} \rightarrow I$ be a homomorphism of complexes representing $\operatorname{Tr}_{B / A}$. We know that the homomorphism $\psi: R_{B} \rightarrow \operatorname{Hom}_{A}(B, I)$ given by the formula $\psi(\beta)(b)=\tau(b \beta)$ is a quasiisomorphism.

Since $R_{A^{\prime}} \cong A^{\prime} \otimes_{A} I \otimes_{A} A^{\prime}$ there is a quasi-isomorphism $A^{\prime} \otimes_{A} I \otimes_{A} A^{\prime} \rightarrow I^{\prime}$, and using it we can assume that $\tau^{\prime}=1 \otimes \tau \otimes 1$ as morphisms

$$
R_{B^{\prime}}=A^{\prime} \otimes_{A} R_{B} \otimes_{A} A^{\prime} \rightarrow A^{\prime} \otimes_{A} I \otimes_{A} A^{\prime} \rightarrow I^{\prime} .
$$

Thus, we get a commutative diagram

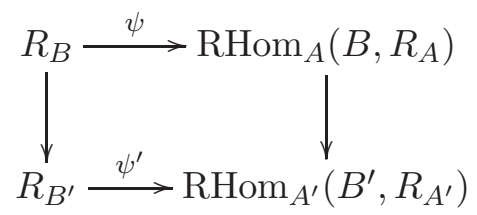

in $\mathrm{D}\left(\operatorname{Mod} A^{\mathrm{e}}\right)$. Applying the base change $-\otimes_{A^{\mathrm{e}}}\left(A^{\prime}\right)^{\mathrm{e}}$ to the diagram we conclude that $\psi^{\prime}=1 \otimes \psi \otimes 1$. So it is an isomorphism.

By symmetry $R_{B^{\prime}} \rightarrow \operatorname{RHom}_{\left(A^{\prime}\right)^{\mathrm{op}}}\left(B^{\prime}, R_{A^{\prime}}\right)$ is also an isomorphism.

Next we have to show that the diagram

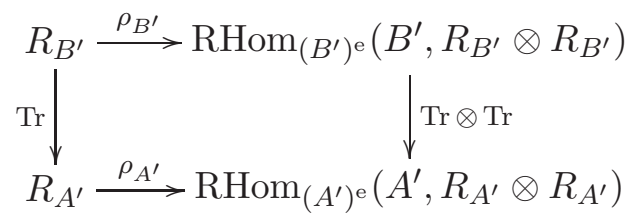

is commutative. This is true since (6.4) was obtained by applying $-\otimes_{A^{\mathrm{e}}}\left(A^{\prime}\right)^{\mathrm{e}}$ to the following commutative diagram.

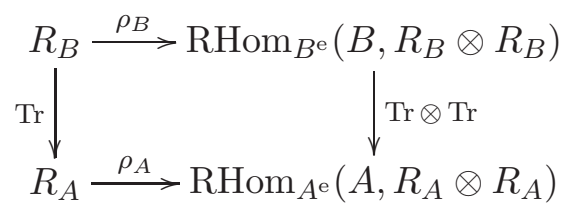

If a $\mathbb{k}$-algebra $A$ has an Auslander rigid dualizing complex $R$, then we write $\operatorname{Cdim}_{A}:=\operatorname{Cdim}_{R ; A}$ for this preferred dimension function.

We finish this section with a digression from our main theme, to present the following corollary to Theorem 6.2. 


\section{DifFERENTIAL ALGEBRAS}

Corollary 6.5. Suppose char $\mathbb{k}=0, A$ is the $n$th Weyl algebra over $\mathbb{k}$ and $D$ is its total ring of fractions, i.e. the $n$th Weyl division ring. Then $D[2 n]$ is an Auslander rigid dualizing complex over $D$, and hence the canonical dimension of $D$ is $\operatorname{Cdim}_{D} D=2 n$.

Proof. By [Ye00], $A[2 n]$ is a rigid Auslander dualizing complex over $A$. Now use Theorem 6.2 with $A^{\prime}:=D$.

We see that unlike the Gelfand-Kirillov dimension GKdim, which cannot distinguish between the various Weyl division rings ( $\operatorname{since} \operatorname{GKdim} D=\infty$ ), the canonical dimension is an intrinsic invariant of $D$ that does recover the number $n$. Moreover, this fact can be expressed as a 'classical' formula, namely

$$
\operatorname{Ext}_{D \otimes \mathrm{D}^{\text {op }}}^{i}(D, D \otimes D) \cong \begin{cases}D & \text { if } i=2 n \\ 0 & \text { otherwise }\end{cases}
$$

\section{Perverse modules and the Auslander condition}

In this section we discuss t-structures on the derived category $\mathrm{D}_{\mathrm{f}}^{\mathrm{b}}(\operatorname{Mod} A)$. We begin by recalling the definition of a t-structure and its basic properties, following [KS90, ch. X].

Definition 7.1. Suppose that $D$ is a triangulated category and $D \leqslant 0, D \geqslant 0$ are two full subcategories. Let $\mathrm{D}^{\leqslant n}:=\mathrm{D}^{\leqslant 0}[-n]$ and $\mathrm{D}^{\geqslant n}:=\mathrm{D}^{\geqslant 0}[-n]$. We say that $\left(\mathrm{D}^{\leqslant 0}, \mathrm{D}^{\geqslant 0}\right)$ is a $t$-structure on $\mathrm{D}$ if:

(i) $\mathrm{D}^{\leqslant-1} \subset \mathrm{D}^{\leqslant 0}$ and $\mathrm{D} \geqslant 1 \subset \mathrm{D} \geqslant 0$;

(ii) $\operatorname{Hom}_{\mathrm{D}}(M, N)=0$ for $M \in \mathrm{D}^{\leqslant 0}$ and $N \in \mathrm{D} \geqslant 1$;

(iii) for any $M \in \mathrm{D}$, there is a distinguished triangle

$$
M^{\prime} \rightarrow M \rightarrow M^{\prime \prime} \rightarrow M^{\prime}[1]
$$

in $\mathrm{D}$ with $M^{\prime} \in \mathrm{D}^{\leqslant 0}$ and $M^{\prime \prime} \in \mathrm{D}^{\geqslant 1}$.

When these conditions are satisfied we define the heart of $\mathrm{D}$ to be the full subcategory $\mathrm{D}^{0}:=\mathrm{D}^{\leqslant 0} \cap \mathrm{D} \geqslant 0$.

Given a t-structure there are truncation functors $\tau^{\leqslant n}: \mathrm{D} \rightarrow \mathrm{D}^{\leqslant n}$ and $\tau^{\geqslant n}: \mathrm{D} \rightarrow \mathrm{D}^{\geqslant n}$, and functorial morphisms $\tau^{\leqslant n} M \rightarrow M, M \rightarrow \tau^{\geqslant n} M$ and $\tau^{\geqslant n+1} M \rightarrow\left(\tau^{\leqslant n} M\right)[1]$ such that

$$
\tau^{\leqslant n} M \rightarrow M \rightarrow \tau^{\geqslant n+1} M \rightarrow\left(\tau^{\leqslant n} M\right)[1]
$$

is a distinguished triangle in $\mathrm{D}$. One shows that the heart $\mathrm{D}^{0}$ is an abelian category, and the functor

$$
\mathrm{H}^{0}:=\tau^{\leqslant 0} \tau^{\geqslant 0} \cong \tau^{\geqslant 0} \tau^{\leqslant 0}: \mathrm{D} \rightarrow \mathrm{D}^{0}
$$

is a cohomological functor.

Example 7.2. Let $A$ be a left noetherian ring. The standard $t$-structure on $\mathrm{D}_{\mathrm{f}}^{\mathrm{b}}(\operatorname{Mod} A)$ is

$$
\mathrm{D}_{\mathrm{f}}^{\mathrm{b}}(\operatorname{Mod} A)^{\leqslant 0}:=\left\{M \in \mathrm{D}_{\mathrm{f}}^{\mathrm{b}}(\operatorname{Mod} A) \mid \mathrm{H}^{j} M=0 \text { for all } j>0\right\}
$$

and

For a complex

$$
\mathrm{D}_{\mathrm{f}}^{\mathrm{b}}(\operatorname{Mod} A)^{\geqslant 0}:=\left\{M \in \mathrm{D}_{\mathrm{f}}^{\mathrm{b}}(\operatorname{Mod} A) \mid \mathrm{H}^{j} M=0 \text { for all } j<0\right\} .
$$

$$
M=\left(\cdots \rightarrow M^{n} \stackrel{\mathrm{d}^{n}}{\longrightarrow} M^{n+1} \rightarrow \cdots\right)
$$

the truncations are

$$
\tau^{\leqslant n} M=\left(\cdots \rightarrow M^{n-2} \rightarrow M^{n-1} \rightarrow \operatorname{Ker}\left(\mathrm{d}^{n}\right) \rightarrow 0 \rightarrow \cdots\right)
$$




\section{A. YeKutieli And J. J. Zhang}

and

$$
\tau^{\geqslant n} M=\left(\cdots \rightarrow 0 \rightarrow \operatorname{Coker}\left(\mathrm{d}^{n-1}\right) \rightarrow M^{n+1} \rightarrow M^{n+2} \rightarrow \cdots\right) .
$$

The heart $\mathrm{D}_{\mathrm{f}}^{\mathrm{b}}(\operatorname{Mod} A)^{0}$ is equivalent to $\operatorname{Mod}_{\mathrm{f}} A$.

Other t-structures on $\mathrm{D}_{\mathrm{f}}^{\mathrm{b}}(\operatorname{Mod} A)$ are referred to as perverse t-structures, and the notation $\left({ }^{p} \mathrm{D}_{\mathrm{f}}^{\mathrm{b}}(\operatorname{Mod} A)^{\leqslant 0},{ }^{p} \mathrm{D}_{\mathrm{f}}^{\mathrm{b}}(\operatorname{Mod} A)^{\geqslant 0}\right)$ is used. The letter ' $p$ ' stands for 'perverse', but often it will also signify a specific perversity function (see below).

Now suppose for $i=1,2$ we are given triangulated categories $\mathrm{D}_{i}$, endowed with t-structures $\left(\mathrm{D}_{i}^{\leqslant 0}, \mathrm{D}_{i}^{\geqslant 0}\right)$. An exact functor $F: \mathrm{D}_{1} \rightarrow \mathrm{D}_{2}$ is called $t$-exact if $F\left(\mathrm{D}_{1}^{\leqslant 0}\right) \subset \mathrm{D}_{2}^{\leqslant 0}$ and $F\left(\mathrm{D}_{1}^{\geqslant 0}\right) \subset \mathrm{D}_{2}^{\geqslant 0}$. The functor $F: \mathrm{D}_{1}^{0} \rightarrow \mathrm{D}_{2}^{0}$ between these abelian categories is then exact. To apply this definition to a contravariant functor $F$ we note that $\left(\left(D^{\geqslant 0}\right)^{\text {op }},\left(D^{\leqslant 0}\right)^{\text {op }}\right)$ is a t-structure on the opposite category $\mathrm{D}^{\mathrm{op}}$. A contravariant triangle functor $F: \mathrm{D}_{1} \rightarrow \mathrm{D}_{2}$ is called t-exact if $F\left(\mathrm{D}_{1}^{\leqslant 0}\right) \subset \mathrm{D}_{2}^{\geqslant 0}$ and $F\left(\mathrm{D}_{1}^{\geqslant 0}\right) \subset \mathrm{D}_{2}^{\leqslant 0}$.

Example 7.3. Let $A$ be a left noetherian $\mathbb{k}$-algebra and let $B$ be a right noetherian $\mathbb{k}$-algebra. Suppose that we are given a dualizing complex $R$ over $(A, B)$, and let $\mathrm{D}$ and $\mathrm{D}^{\mathrm{op}}$ be the duality functors that $R$ induces; see Definitions 4.1 and 4.5. Put the standard t-structure on $D_{\mathrm{f}}^{\mathrm{b}}\left(\operatorname{Mod} B^{\mathrm{op}}\right)$ (see Example 7.2). Define subcategories

$$
{ }^{p} \mathrm{D}_{\mathrm{f}}^{\mathrm{b}}(\operatorname{Mod} A) \leqslant 0:=\left\{M \in \mathrm{D}_{\mathrm{f}}^{\mathrm{b}}(\operatorname{Mod} A) \mid \mathrm{D} M \in \mathrm{D}_{\mathrm{f}}^{\mathrm{b}}\left(\operatorname{Mod} B^{\mathrm{op}}\right) \geqslant 0\right\}
$$

and

$$
{ }^{p} \mathrm{D}_{\mathrm{f}}^{\mathrm{b}}(\operatorname{Mod} A)^{\geqslant 0}:=\left\{M \in \mathrm{D}_{\mathrm{f}}^{\mathrm{b}}(\operatorname{Mod} A) \mid \mathrm{D} M \in \mathrm{D}_{\mathrm{f}}^{\mathrm{b}}\left(\operatorname{Mod} B^{\mathrm{op}}\right)^{\leqslant 0}\right\} .
$$

Since $\mathrm{D}: \mathrm{D}_{\mathrm{f}}^{\mathrm{b}}(\operatorname{Mod} A) \rightarrow \mathrm{D}_{\mathrm{f}}^{\mathrm{b}}\left(\operatorname{Mod} B^{\mathrm{op}}\right)$ is a duality it follows that

$$
\left({ }^{p} \mathrm{D}_{\mathrm{f}}^{\mathrm{b}}(\operatorname{Mod} A)^{\leqslant 0},{ }^{p} \mathrm{D}_{\mathrm{f}}^{\mathrm{b}}(\operatorname{Mod} A)^{\geqslant 0}\right)
$$

is a t-structure on $\mathrm{D}_{\mathrm{f}}^{\mathrm{b}}(\operatorname{Mod} A)$, which we call the perverse $t$-structure induced by $R$. The functors $\mathrm{D}$ and $\mathrm{D}^{\mathrm{op}}$ are t-exact, and

$$
\mathrm{D}:{ }^{p} \mathrm{D}_{\mathrm{f}}^{\mathrm{b}}(\operatorname{Mod} A)^{0} \rightarrow \mathrm{D}_{\mathrm{f}}^{\mathrm{b}}\left(\operatorname{Mod} B^{\mathrm{op}}\right)^{0} \approx \operatorname{Mod}_{\mathrm{f}} B^{\mathrm{op}}
$$

is a duality of abelian categories.

Definition 7.4. Suppose that $A$ is a noetherian $\mathbb{k}$-algebra with rigid dualizing complex $R_{A}$. The perverse t-structure induced on $\mathrm{D}_{\mathrm{f}}^{\mathrm{b}}(\operatorname{Mod} A)$ by $R_{A}$ is called the rigid perverse $t$-structure. An object $M \in{ }^{p} \mathrm{D}_{\mathrm{f}}^{\mathrm{b}}(\operatorname{Mod} A)^{0}$ is called a perverse $A$-module.

In the remainder of this section we concentrate on another method of producing t-structures on $\mathrm{D}_{\mathrm{f}}^{\mathrm{b}}(\operatorname{Mod} A)$. This method is of a geometric nature, and closely resembles the t-structures that originally appeared in [BBD81].

A perversity is a function $p: \mathbb{Z} \rightarrow \mathbb{Z}$ satisfying $p(i)-1 \leqslant p(i+1) \leqslant p(i)$. We call the function $p(i)=0$ the trivial perversity, and the function $p(i)=-i$ is called the minimal perversity.

Let $A$ be a ring. Fix an exact dimension function $\operatorname{dim}$ on $\operatorname{Mod} A$ (see Definition 4.8). For an integer $i$ let $\mathrm{M}_{i}(\operatorname{dim})$ be the full subcategory of $\operatorname{Mod} A$ consisting of the modules $M$ with $\operatorname{dim} M \leqslant i$. The subcategory $\mathrm{M}_{i}(\mathrm{dim})$ is localizing, and there is a functor

$$
\Gamma_{\mathrm{M}_{i}(\operatorname{dim})}: \operatorname{Mod} A \rightarrow \operatorname{Mod} A
$$

defined by

$$
\Gamma_{\mathrm{M}_{i}(\operatorname{dim})} M:=\{m \in M \mid \operatorname{dim} A m \leqslant i\} \subset M .
$$

The functor $\Gamma_{\mathrm{M}_{i}(\operatorname{dim})}$ has a derived functor

$$
\mathrm{R} \Gamma_{\mathrm{M}_{i}(\operatorname{dim})}: \mathrm{D}^{+}(\operatorname{Mod} A) \rightarrow \mathrm{D}^{+}(\operatorname{Mod} A)
$$




\section{DIFFERENTIAL ALGEBRAS}

calculated using injective resolutions. For $M \in \mathrm{D}^{+}(\operatorname{Mod} A)$ the $j$ th cohomology of $M$ with supports in $\mathrm{M}_{i}(\mathrm{dim})$ is defined to be

$$
\mathrm{H}_{\mathrm{M}_{i}(\operatorname{dim})}^{j} M:=\mathrm{H}^{j} \mathrm{R} \Gamma_{\mathrm{M}_{i}(\operatorname{dim})} M
$$

The definition above was introduced in [Ye96, YZ03]. It is based on the following geometric paradigm.

Example 7.5. If $A$ is a commutative noetherian $\operatorname{ring}$ of finite Krull dimension and we set $\operatorname{dim} M:=$ $\operatorname{dim} \operatorname{Supp} M$ for a finite module $M$, then

$$
\mathrm{H}_{\mathrm{M}_{i}(\operatorname{dim})}^{j} M \cong \lim _{\rightarrow} \mathrm{H}_{Z}^{j} M
$$

where $Z$ runs over the closed sets in $\operatorname{Spec} A$ of dimension $\leqslant i$.

Definition 7.6. Let $A$ be a left noetherian ring. Given an exact dimension function $\operatorname{dim}$ on $\operatorname{Mod} A$ and a perversity $p$, define subcategories

$$
{ }^{p} \mathrm{D}_{\mathrm{f}}^{\mathrm{b}}(\operatorname{Mod} A)^{\leqslant 0}:=\left\{M \in \mathrm{D}_{\mathrm{f}}^{\mathrm{b}}(\operatorname{Mod} A) \mid \operatorname{dim} \mathrm{H}^{j} M<i \text { for all } i, j \text { with } j>p(i)\right\}
$$

and

$$
{ }^{p} \mathrm{D}_{\mathrm{f}}^{\mathrm{b}}(\operatorname{Mod} A)^{\geqslant 0}:=\left\{M \in \mathrm{D}_{\mathrm{f}}^{\mathrm{b}}(\operatorname{Mod} A) \mid \mathrm{H}_{\mathrm{M}_{i}(\operatorname{dim})}^{j} M=0 \text { for all } i, j \text { with } j<p(i)\right\}
$$

of $\mathrm{D}_{\mathrm{f}}^{\mathrm{b}}(\operatorname{Mod} A)$.

Example 7.7. Suppose $\operatorname{dim}$ is any exact dimension function such that $\operatorname{dim} M>-\infty$ for all $M \neq 0$. Take the trivial perversity $p(i)=0$. Then

$$
{ }^{p} \mathrm{D}_{\mathrm{f}}^{\mathrm{b}}(\operatorname{Mod} A)^{\leqslant 0}=\mathrm{D}_{\mathrm{f}}^{\mathrm{b}}(\operatorname{Mod} A)^{\leqslant 0}
$$

and

$$
{ }^{p} \mathrm{D}_{\mathrm{f}}^{\mathrm{b}}(\operatorname{Mod} A)^{\geqslant 0}=\mathrm{D}_{\mathrm{f}}^{\mathrm{b}}(\operatorname{Mod} A)^{\geqslant 0},
$$

namely the standard t-structure on $\mathrm{D}_{\mathrm{f}}^{\mathrm{b}}(\operatorname{Mod} A)$.

The following lemma is straightforward.

Lemma 7.8. In the situation of Definition 7.6 , let $p$ be the minimal perversity, namely $p(i)=-i$, and let $M \in \mathrm{D}_{\mathrm{f}}^{\mathrm{b}}(\operatorname{Mod} A)$. Then:

(1) $M \in{ }^{p} \mathrm{D}_{\mathrm{f}}^{\mathrm{b}}(\operatorname{Mod} A)^{\leqslant 0}$ if and only if $\operatorname{dim} \mathrm{H}^{-i} M \leqslant i$ for all $i$;

(2) $M \in{ }^{p} \mathrm{D}_{\mathrm{f}}^{\mathrm{b}}(\operatorname{Mod} A)^{\geqslant 0}$ if and only if $\mathrm{H}_{\mathrm{M}_{i}(\operatorname{dim})}^{j} M=0$ for all $j<-i$, if and only if $\mathrm{R}_{\mathrm{M}_{i}(\operatorname{dim})} M \in$ $\mathrm{D}_{\mathrm{f}}^{\mathrm{b}}(\operatorname{Mod} A)^{\geqslant-i}$ for all $i$.

Recall that if $R$ is an Auslander dualizing complex over the rings $(A, B)$ then the canonical dimension $\operatorname{Cdim}_{R}$ (Definition 4.9) is an exact dimension function on $\operatorname{Mod} A$.

Theorem 7.9. Let $A$ be a left noetherian $\mathbb{k}$-algebra and $B$ a right noetherian $\mathbb{k}$-algebra. Suppose $R$ is an Auslander dualizing complex over $(A, B)$. Let $\operatorname{dim}$ be the canonical dimension function $\operatorname{Cdim}_{R ; A}$ on $\operatorname{Mod} A$, and let $p$ be the minimal perversity $p(i)=-i$. Then:

(1) the pair

$$
\left({ }^{p} \mathrm{D}_{\mathrm{f}}^{\mathrm{b}}(\operatorname{Mod} A)^{\leqslant 0},{ }^{p} \mathrm{D}_{\mathrm{f}}^{\mathrm{b}}(\operatorname{Mod} A)^{\geqslant 0}\right)
$$

from Definition 7.6 is a t-structure on $\mathrm{D}_{\mathrm{f}}^{\mathrm{b}}(\operatorname{Mod} A)$;

(2) put on $\mathrm{D}_{\mathrm{f}}^{\mathrm{b}}\left(\operatorname{Mod} B^{\mathrm{op}}\right)$ the standard t-structure, then the duality functors $\mathrm{D}$ and $\mathrm{D}^{\mathrm{op}}$ determined by $R$ (see Definition 4.5 ) are t-exact. 


\section{A. YeKutieli And J. J. Zhang}

Proof. In the proof we use the abbreviations $\mathrm{D}(A):=\mathrm{D}_{\mathrm{f}}^{\mathrm{b}}(\operatorname{Mod} A)$, etc.

If $M \in{ }^{p} \mathrm{D}(A)^{\leqslant-1}$, then $M[-1] \in{ }^{p} \mathrm{D}(A)^{\leqslant 0}$. By Lemma $7.8(1)$ we get $\operatorname{dim} \mathrm{H}^{-i}(M[-1]) \leqslant i$. Changing indices, we get $\operatorname{dim} \mathrm{H}^{-i} M \leqslant i-1 \leqslant i$. Again using Lemma 7.8(1), we see that the first part of condition (i) of Definition 7.1 is verified. The second part of condition (i) is verified similarly using Lemma 7.8(2).

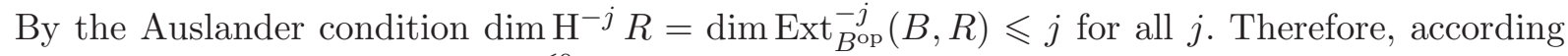
to Lemma 7.8(1) we get $R \in{ }^{p} \mathrm{D}(A)^{\leqslant 0}$. On the other hand, since

$$
\mathrm{H}_{\mathrm{M}_{i}(\operatorname{dim})}^{j} R \cong \underset{\mathfrak{a} \in \mathfrak{F}}{\lim _{\longrightarrow}} \operatorname{Ext}_{A}^{j}(A / \mathfrak{a}, R),
$$

where $\mathfrak{F}$ is the Gabriel filter of left ideals corresponding to $\mathrm{M}_{i}(\operatorname{dim})$, the Auslander condition and Lemma 7.8(2) imply that $R \in{ }^{p} \mathrm{D}(A)^{\geqslant 0}$.

Let $M^{\prime} \rightarrow M \rightarrow M^{\prime \prime} \rightarrow M^{\prime}[1]$ be a distinguished triangle in $\mathrm{D}(A)$. Since dim is exact, and using the criterion in Lemma $7.8(1)$, we see that if $M^{\prime}$ and $M^{\prime \prime}$ are in ${ }^{p} \mathrm{D}(A)^{\leqslant 0}$, then so is $M$. Likewise, applying the functor $\mathrm{H}_{\mathrm{M}_{i}(\operatorname{dim})}^{j}$ to this triangle and using Lemma 7.8(2) it follows that if $M^{\prime}$ and $M^{\prime \prime}$ are in ${ }^{p} \mathrm{D}(A)^{\geqslant 0}$, then so is $M$.

Suppose that we are given $M \in{ }^{p} \mathrm{D}(A)^{\leqslant 0}, N \in{ }^{p} \mathrm{D}(A)^{\geqslant 1}$ and a morphism $\phi: M \rightarrow N$. In order to prove that $\phi=0$ we first assume that $M$ is a single finite module, concentrated in some degree $-l$, with $l \geqslant 0$ and $\operatorname{dim} M \leqslant l$. Then $\phi$ factors through $M \stackrel{\phi^{\prime}}{\longrightarrow} \mathrm{R}_{\mathrm{M}_{l}(\operatorname{dim})} N \rightarrow N$. Now $M \in \mathrm{D}(A) \leqslant-l$ and, by Lemma 7.8(2), $\mathrm{R} \Gamma_{\mathrm{M}_{l}(\operatorname{dim})} N \in \mathrm{D}(A) \geqslant-l+1$; hence $\phi^{\prime}=0$. Next let us consider the general case. Let $\mathrm{H}^{-l} M$ be the lowest nonzero cohomology of $M$. We have a distinguished triangle

$$
T:=\left(\left(\mathrm{H}^{-l} M\right)[l] \rightarrow M \rightarrow M^{\prime \prime} \rightarrow\left(\mathrm{H}^{-l} M\right)[l+1]\right)
$$

where $M^{\prime \prime}$ is the standard truncation of $M$. According to Lemma 7.8(1) we have $\operatorname{dim} \mathrm{H}^{-l} M \leqslant$ $l$, so by the previous argument the composition $\left(\mathrm{H}^{-l} M\right)[l] \rightarrow M \stackrel{\phi}{\rightarrow} N$ is zero. So applying $\operatorname{Hom}_{\mathrm{D}(\operatorname{Mod} A)}(-, N)$ to the triangle $T$, we conclude that $\phi$ comes from some morphism $\phi^{\prime \prime}: M^{\prime \prime} \rightarrow N$. Since $M^{\prime \prime} \in{ }^{p} \mathrm{D}(A)^{\leqslant 0}$ and by induction on the number of nonvanishing cohomologies we have $\phi^{\prime \prime}=0$. Therefore, condition (ii) is verified.

Next suppose that $M \in \mathrm{D}\left(B^{\mathrm{op}}\right)^{\leqslant 0}$. In order to prove that $\mathrm{D}^{\mathrm{op}} M \in{ }^{p} \mathrm{D}(A)^{\geqslant 0}$ we can assume that $M$ is a single finite $B^{\mathrm{op}}$-module, concentrated in degree $-l$ for some $l \geqslant 0$. By [YZ03, Proposition 5.2] and its proof we deduce that

$$
\begin{aligned}
\mathrm{R}_{\mathrm{M}_{i}(\operatorname{dim})} \mathrm{D}^{\mathrm{op}} M & =\mathrm{R}_{\mathrm{M}_{i}(\operatorname{dim})} \operatorname{RHom}_{B^{\mathrm{op}}}(M, R) \\
& \cong \mathrm{RHom}_{B^{\mathrm{op}}}\left(M, \mathrm{R} \Gamma_{\mathrm{M}_{i}(\operatorname{dim})} R\right) .
\end{aligned}
$$

As we saw above, the Auslander condition implies that $\mathrm{R} \Gamma_{\mathrm{M}_{i}(\operatorname{dim})} R \in \mathrm{D}\left(B^{\mathrm{op}}\right) \geqslant-i$. However, $M \in$ $\mathrm{D}\left(B^{\mathrm{op}}\right) \leqslant-l$, and hence

$$
\mathrm{RHom}_{B^{\mathrm{op}}}\left(M, \mathrm{R}_{\mathrm{M}_{i}(\operatorname{dim})} R\right) \in \mathrm{D}(A)^{\geqslant l-i} \subset \mathrm{D}(A)^{\geqslant-i} .
$$

Now the criterion in Lemma 7.8(2) tells us that $\mathrm{D}^{\mathrm{op}} M \in{ }^{p} \mathrm{D}(A) \geqslant 0$.

Let $M \in \mathrm{D}\left(B^{\mathrm{op}}\right) \geqslant 0$. We wish to prove that $\mathrm{D}^{\mathrm{op}} M \in{ }^{p} \mathrm{D}(A)^{\leqslant 0}$. To do so we can assume that $M$ is a single finite module, concentrated in some degree $l \geqslant 0$. Then for every $i, \mathrm{H}^{-i} \mathrm{D}^{\mathrm{op}} M=\operatorname{Ext}_{B^{\mathrm{op}}}^{-i}(M, R)$ has $\operatorname{dim} \mathrm{H}^{-i} \mathrm{D}^{\mathrm{op}} M \leqslant i$. Now apply Lemma 7.8(1).

At this point we know that $\mathrm{D}^{\mathrm{op}}\left(\mathrm{D}\left(B^{\mathrm{op}}\right)^{\leqslant 0}\right) \subset{ }^{p} \mathrm{D}(A)^{\geqslant 0}$ and $\mathrm{D}^{\mathrm{op}}\left(\mathrm{D}\left(B^{\mathrm{op}}\right) \geqslant 0\right) \subset{ }^{p} \mathrm{D}(A)^{\leqslant 0}$. Let $M \in \mathrm{D}(A)$ be an arbitrary complex, and consider the distinguished triangle

$$
\tau^{\leqslant-1} \mathrm{D} M \rightarrow \mathrm{D} M \rightarrow \tau^{\geqslant 0} \mathrm{D} M \rightarrow\left(\tau^{\leqslant-1} \mathrm{D} M\right)[1]
$$

in $\mathrm{D}\left(B^{\mathrm{op}}\right)$ obtained from the standard t-structure there. Applying $\mathrm{D}^{\mathrm{op}}$ we obtain a distinguished 


\section{DifFERENTIAL ALGEBRAS}

triangle

$$
M^{\prime} \rightarrow M \rightarrow M^{\prime \prime} \rightarrow M^{\prime}[1]
$$

in $\mathrm{D}(A)$, where $M^{\prime}:=\mathrm{D}^{\mathrm{op}} \tau^{\geqslant 0} \mathrm{D} M$ and $M^{\prime \prime}:=\mathrm{D}^{\mathrm{op}} \tau^{\leqslant-1} \mathrm{D} M$. This proves that condition (iii) is fulfilled, so we have a t-structure on $\mathrm{D}(A)$, and also that the functor $\mathrm{D}^{\mathrm{op}}$ is t-exact.

To finish the proof we invoke [KS90, Corollary 10.1.18], which tells us that D is also t-exact.

Problem 7.10. Let $A$ be a left noetherian ring. Find necessary and sufficient conditions on a dimension function $\operatorname{dim}$ on $\operatorname{Mod} A$, and on a perversity function $p$, such that Theorem 7.9(1) holds.

Remark 7.11. The idea for Definition 7.6 comes from [KS90, p. 438, Exercise X.2]. In his recent paper Kashiwara [Ka03] considered similar t-structures. In particular, his results imply that when $A=B$ is a commutative finitely generated $\mathbb{k}$-algebra, and $R$ is the rigid dualizing complex of $A$, then Theorem 7.9 holds for any perversity function $p$. (Note that here canonical dimension coincides with Krull dimension.) In part (2) of the theorem one has to put the perverse t-structure determined by the dual perversity $p^{*}(i):=-i-p(i)$ on $\mathrm{D}_{\mathrm{f}}^{\mathrm{b}}\left(\operatorname{Mod} A^{\mathrm{op}}\right)$.

\section{The rigid dualizing complex of a differential $\mathbb{k}$-algebra}

We begin this section with the following consequence of previous work.

TheOREm 8.1. Let $A$ be a differential $\mathbb{k}$-algebra of finite type. Then $A$ has an Auslander rigid dualizing complex $R_{A}$. For a finite $A$-module $M$ the canonical dimension $\operatorname{Cdim} M$ coincides with the Gelfand-Kirillov dimension GKdim $M$.

Proof. According to Theorem 3.1, $A$ has a nonnegative exhaustive filtration $G=\left\{G_{i} A\right\}$ such that $\mathrm{gr}^{G} A$ is a commutative, finitely generated, connected graded $\mathbb{k}$-algebra. Now use [YZ99, Corollary $6.9]$.

Recall that a ring homomorphism $f: A \rightarrow B$ is called finite centralizing if there exist elements $b_{1}, \ldots, b_{n} \in B$ that commute with all elements of $A$ and $B=\sum_{i} A b_{i}$.

Proposition 8.2. Let $A$ be a differential $\mathbb{k}$-algebra of finite type and $f: A \rightarrow B$ a finite centralizing homomorphism. Then $B$ is also a differential $\mathbb{k}$-algebra of finite type, and the rigid trace $\operatorname{Tr}_{B / A}$ : $R_{B} \rightarrow R_{A}$ exists.

Proof. By Theorem 3.1 we can find a differential $\mathbb{k}$-filtration of finite type $F=\left\{F_{i} A\right\}$ of $A$ such that $\operatorname{gr}^{F} A$ is connected. By [YZ99, Lemma 6.13] and its proof there is a filtration $F=\left\{F_{i} B\right\}$ of $B$ such that $\operatorname{gr}^{F} B$ is connected, $f\left(F_{i} A\right) \subset F_{i} B$ and $\operatorname{gr}^{F}(f): \operatorname{gr}^{F} A \rightarrow \operatorname{gr}^{F} B$ is a finite centralizing homomorphism. It follows that $\operatorname{gr}^{F} B$ is finite over its center, so $B$ is a differential $\mathbb{k}$-algebra of finite type. By [YZ99, Theorem 6.17] the rigid trace $\operatorname{Tr}_{B / A}: R_{B} \rightarrow R_{A}$ exists.

Let $A$ be a differential $\mathbb{k}$-algebra of finite type with rigid dualizing complex $R_{A}$. The derived category $\mathrm{D}_{\mathrm{f}}^{\mathrm{b}}(\operatorname{Mod} A)$ has on it the rigid perverse t-structure induced by $R_{A}$, whose heart is the category of perverse $A$-modules ${ }^{p} \mathrm{D}_{\mathrm{f}}^{\mathrm{b}}(\operatorname{Mod} A)^{0}$. See Definition 7.4.

Proposition 8.3. Let $A \rightarrow B$ be a finite centralizing homomorphism between two differential $\mathbb{k}$-algebras of finite type. Denote by $\operatorname{rest}_{B / A}: \mathrm{D}(\operatorname{Mod} B) \rightarrow \mathrm{D}(\operatorname{Mod} A)$ the restriction of scalars functor.

(1) Let $M \in \mathrm{D}_{\mathrm{f}}^{\mathrm{b}}(\operatorname{Mod} B)$. Then $M \in{ }^{p} \mathrm{D}_{\mathrm{f}}^{\mathrm{b}}(\operatorname{Mod} B)^{0}$ if and only if $\operatorname{rest}_{B / A} M \in{ }^{p} \mathrm{D}_{\mathrm{f}}^{\mathrm{b}}(\operatorname{Mod} A)^{0}$.

(2) If $A \rightarrow B$ is surjective, then the functor

$$
\operatorname{rest}_{B / A}:{ }^{p} \mathrm{D}_{\mathrm{f}}^{\mathrm{b}}(\operatorname{Mod} B)^{0} \rightarrow{ }^{p} \mathrm{D}_{\mathrm{f}}^{\mathrm{b}}(\operatorname{Mod} A)^{0}
$$

is fully faithful. 


\section{A. YeKutieli AND J. J. Zhang}

Proof. (1) Define the duality functors $\mathrm{D}_{A}:=\mathrm{RHom}_{A}\left(-, R_{A}\right)$ and $\mathrm{D}_{B}:=\mathrm{RHom}_{B}\left(-, R_{B}\right)$. According to [YZ99, Proposition 3.9(1)] the trace $\operatorname{Tr}_{B / A}: R_{B} \rightarrow R_{A}$ gives rise to a commutative diagram

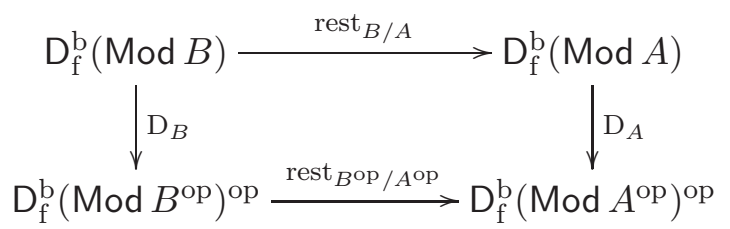

in which the vertical arrows are equivalences. By definition $M \in{ }^{p} \mathrm{D}_{\mathrm{f}}^{\mathrm{b}}(\operatorname{Mod} B)^{0}$ if and only if $\mathrm{H}^{i} \mathrm{D}_{B} M=0$ for all $i \neq 0$. Likewise, rest $_{B / A} M \in{ }^{p} \mathrm{D}_{\mathrm{f}}^{\mathrm{b}}(\operatorname{Mod} A)^{0}$ if and only if $\mathrm{H}^{i} \mathrm{D}_{A}$ rest $_{B / A} M=0$ for all $i \neq 0$. However,

$$
\mathrm{H}^{i} \mathrm{D}_{A} \operatorname{rest}_{B / A} M \cong \operatorname{rest}_{B} \text { op } / A^{\text {op }} \mathrm{H}^{i} \mathrm{D}_{B} M .
$$

(2) In view of (1) we have a commutative diagram

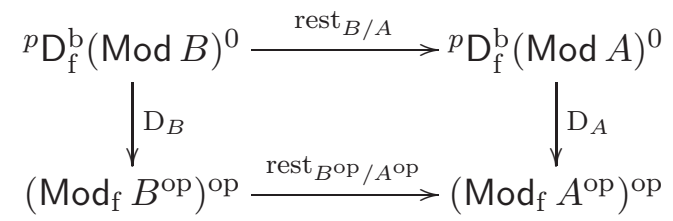

where the vertical arrows are equivalences. The lower horizontal arrow is a full embedding, since it identifies $\operatorname{Mod}_{\mathrm{f}} B^{\mathrm{op}}$ with the full subcategory of $\operatorname{Mod}_{\mathrm{f}} A^{\text {op }}$ consisting of modules annihilated by $\operatorname{Ker}\left(A^{\mathrm{op}} \rightarrow B^{\mathrm{op}}\right)$. Hence, the top horizontal arrow is fully faithful.

Lemma 8.4. Suppose that $A$ and $B$ are $\mathbb{k}$-algebras, $M, M^{\prime} \in \mathrm{D}^{\mathrm{b}}\left(\operatorname{Mod} A^{\mathrm{e}}\right)$ and $N, N^{\prime} \in \mathrm{D}^{\mathrm{b}}\left(\operatorname{Mod} B^{\mathrm{e}}\right)$. Then there is a functorial morphism

$$
\mu: \operatorname{RHom}_{A}\left(M, M^{\prime}\right) \otimes \operatorname{RHom}_{B}\left(N, N^{\prime}\right) \rightarrow \operatorname{RHom}_{A \otimes B}\left(M \otimes N, M^{\prime} \otimes N^{\prime}\right)
$$

in $\mathrm{D}\left(\operatorname{Mod}(A \otimes B)^{\mathrm{e}}\right)$. If $A$ and $B$ are left noetherian and all the modules $\mathrm{H}^{p} M$ and $\mathrm{H}^{p} N$ are finite, then $\mu$ is an isomorphism.

Proof. Choose projective resolutions $P \rightarrow M$ and $Q \rightarrow N$ over $A^{\mathrm{e}}$ and $B^{\mathrm{e}}$, respectively. So $P \otimes Q \rightarrow$ $M \otimes N$ is a projective resolution over $(A \otimes B)^{\mathrm{e}}$, and we get a map of complexes

$$
\mu: \operatorname{Hom}_{A}\left(P, M^{\prime}\right) \otimes \operatorname{Hom}_{B}\left(Q, N^{\prime}\right) \rightarrow \operatorname{Hom}_{A \otimes B}\left(P \otimes Q, M^{\prime} \otimes N^{\prime}\right)
$$

over $(A \otimes B)^{\mathrm{e}}$.

Now assume the finiteness of the cohomologies. To prove that $\mu$ is a quasi-isomorphism we might as well forget the right module structures. Choose resolutions $P_{\mathrm{f}} \rightarrow M$ and $Q_{\mathrm{f}} \rightarrow N$ by complexes of finite projective modules over $A$ and $B$, respectively. We obtain a commutative diagram

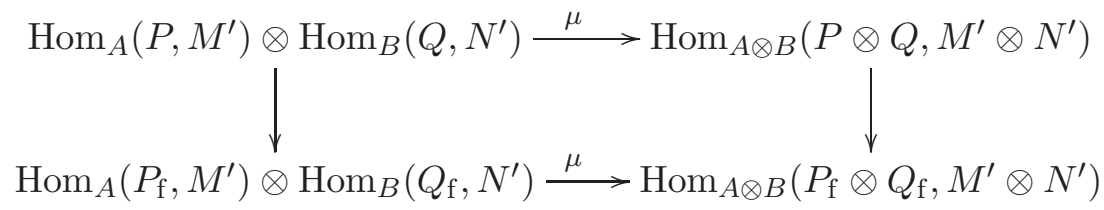

in which the vertical arrows are quasi-isomorphism and the bottom arrow is an isomorphism of complexes.

Recall that by Proposition 2.9 the tensor product of two differential $\mathbb{k}$-algebras of finite type is also a differential $\mathbb{k}$-algebra of finite type. 


\section{DifFERENTIAL ALGEBRAS}

Theorem 8.5. Suppose that $A$ and $B$ are differential $\mathbb{k}$-algebras of finite type. Then the rigid dualizing complexes satisfy

$$
R_{A \otimes B} \cong R_{A} \otimes R_{B}
$$

in $\mathrm{D}\left(\operatorname{Mod}(A \otimes B)^{\mathrm{e}}\right)$.

Proof. We will prove that $R_{A} \otimes R_{B}$ is a rigid dualizing complex over $A \otimes B$.

Consider the Kunneth spectral sequence

$$
\left(\mathrm{H}^{p} R_{A}\right) \otimes\left(\mathrm{H}^{q} R_{B}\right) \Rightarrow \mathrm{H}^{p+q}\left(R_{A} \otimes R_{B}\right) .
$$

Since $A \otimes B$ is noetherian it follows that $\mathrm{H}^{p+q}\left(R_{A} \otimes R_{B}\right)$ is a finite $(A \otimes B)$-module on both sides.

From Lemma 8.4 we see that the canonical morphism

$$
A \otimes B \rightarrow \operatorname{RHom}_{A \otimes B}\left(R_{A} \otimes R_{B}, R_{A} \otimes R_{B}\right)
$$

in $\mathrm{D}\left(\operatorname{Mod}(A \otimes B)^{\mathrm{e}}\right)$ is an isomorphism. Likewise for $\mathrm{RHom}_{(A \otimes B) \mathrm{e}}$.

Next using this lemma with $A^{\mathrm{e}}$ and $B^{\mathrm{e}}$, and by the rigidity of $R_{A}$ and $R_{B}$, we get isomorphisms

$$
\begin{aligned}
& \operatorname{RHom}_{(A \otimes B) \mathrm{e}}\left(A \otimes B,\left(R_{A} \otimes R_{B}\right) \otimes\left(R_{A} \otimes R_{B}\right)\right) \\
& \cong \operatorname{RHom}_{A^{\mathrm{e}} \otimes B^{\mathrm{e}}}\left(A \otimes B,\left(R_{A} \otimes R_{A}\right) \otimes\left(R_{B} \otimes R_{B}\right)\right) \\
& \cong \mathrm{RHom}_{A^{\mathrm{e}}}\left(A, R_{A} \otimes R_{A}\right) \otimes \mathrm{RHom}_{B^{\mathrm{e}}}\left(B, R_{B} \otimes R_{B}\right) \\
& \cong R_{A} \otimes R_{B}
\end{aligned}
$$

in $\mathrm{D}\left(\operatorname{Mod}(A \otimes B)^{\mathrm{e}}\right)$.

It remains to prove that the complex $R_{A} \otimes R_{B}$ has finite injective dimension over $A \otimes B$ and over $(A \otimes B)^{\mathrm{op}}$. This turns out to be quite difficult (cf. Corollary 8.6 below). By Theorem 3.1 there is a filtration $F$ of $A$ such that $\operatorname{gr}^{F} A$ is connected, finitely generated and commutative. Let $\widetilde{A}:=$ Rees $^{F} A \subset A[s]$, which is a noetherian connected graded $\mathbb{k}$-algebra. By [YZ99, Theorem 5.13], $\widetilde{A}$ has a balanced dualizing complex $R_{\widetilde{A}} \in \mathrm{D}^{\mathrm{b}}\left(\operatorname{GrMod}(\widetilde{A})^{\mathrm{e}}\right)$. The same holds for $B$ : there is a filtration $G$, a Rees ring $\widetilde{B}:=\operatorname{Rees}^{G} B \subset B[t]$ and a balanced dualizing complex $R_{\widetilde{B}}$. According to [vdB97, Theorem 7.1] the complex $R_{\widetilde{A}} \otimes R_{\widetilde{B}}$ is a balanced dualizing complex over $\widetilde{A} \otimes \widetilde{B}$. In particular, $R_{\widetilde{A}} \otimes R_{\widetilde{B}}$ has finite graded-injective dimension over $\widetilde{A} \otimes \widetilde{B}$.

Now $A \cong \widetilde{A} /(s-1)$, so by [YZ99, Lemma 6.3] the complex

$$
Q:=(A \otimes \widetilde{B}) \otimes \widetilde{A} \otimes \widetilde{B}\left(R_{\widetilde{A}} \otimes R_{\widetilde{B}}\right)
$$

has finite injective dimension over $A \otimes \widetilde{B}$. However, the algebra $A \otimes \widetilde{B}$ is graded (the element $1 \otimes t$ has degree 1$)$, and $Q$ is a complex of graded $(A \otimes \widetilde{B})$-modules. Therefore, $Q$ has finite graded-injective dimension over this graded ring. Applying [YZ99, Lemma 6.3] again (it works for any graded ring, connected or not) we see that

$$
(A \otimes B) \otimes_{A \otimes \widetilde{B}} Q \cong(A \otimes B) \otimes_{\widetilde{A} \otimes \widetilde{B}}\left(R_{\widetilde{A}} \otimes R_{\widetilde{B}}\right)
$$

has finite injective dimension over $A \otimes B$.

According to [YZ99, Theorem 6.2] there is an isomorphism $R_{A} \cong A \otimes_{\widetilde{A}} R_{\widetilde{A}}[-1]$ in $\mathrm{D}(\operatorname{Mod} A)$. Likewise, $R_{B} \cong B \otimes_{\widetilde{B}} R_{\widetilde{B}}[-1]$. Hence,

$$
R_{A} \otimes R_{B} \cong(A \otimes B) \otimes_{\widetilde{A} \otimes \widetilde{B}}\left(R_{\widetilde{A}} \otimes R_{\widetilde{B}}\right)[-2]
$$

has finite injective dimension over $A \otimes B$.

By symmetry $R_{A} \otimes R_{B}$ has finite injective dimension also over $(A \otimes B)^{\mathrm{op}}$. 


\section{A. YeKutieli And J. J. Zhang}

Corollary 8.6. Suppose that $A$ and $B$ are differential $\mathbb{k}$-algebras of finite type, and the complexes $M \in \mathrm{D}_{\mathrm{f}}^{\mathrm{b}}(\operatorname{Mod} A)$ and $N \in \mathrm{D}_{\mathrm{f}}^{\mathrm{b}}(\operatorname{Mod} B)$ have finite injective dimension over $A$ and $B$, respectively. Then $M \otimes N$ has finite injective dimension over $A \otimes B$.

Proof. Let $M^{\vee}:=\operatorname{RHom}_{A}\left(M, R_{A}\right)$ and $N^{\vee}:=\operatorname{RHom}_{B}\left(N, R_{B}\right)$. The complexes $M^{\vee} \in \mathrm{D}_{\mathrm{f}}^{\mathrm{b}}\left(\operatorname{Mod} A^{\text {op }}\right)$ and $N^{\vee} \in \mathrm{D}_{\mathrm{f}}^{\mathrm{b}}\left(\operatorname{Mod} B^{\mathrm{op}}\right.$ ) have finite projective dimension (i.e. they are perfect); see [Ye99, Theorem 4.5]. Since the tensor product of projective modules is projective it follows that $M^{\vee} \otimes N^{\vee} \in$ $\mathrm{D}_{\mathrm{f}}^{\mathrm{b}}\left(\operatorname{Mod}\left(A^{\mathrm{op}} \otimes B^{\mathrm{op}}\right)\right)$ has finite projective dimension. Using Theorem 8.5, and Lemma 8.4 we see that

$$
\mathrm{RHom}_{A \otimes B}\left(M \otimes N, R_{A \otimes B}\right) \cong \mathrm{RHom}_{A \otimes B}\left(M \otimes N, R_{A} \otimes R_{B}\right) \cong M^{\vee} \otimes N^{\vee} .
$$

Applying $\mathrm{RHom}_{A \otimes B}\left(-, R_{A \otimes B}\right)$ to these isomorphisms we get

$$
M \otimes N \cong \operatorname{RHom}_{A \otimes B}\left(M^{\vee} \otimes N^{\vee}, R_{A \otimes B}\right),
$$

so this complex has finite injective dimension.

Problem 8.7. Is there a direct proof of the corollary? Is it true in greater generality, for example, for any two noetherian $\mathbb{k}$-algebras $A$ and $B$ ?

Remark 8.8. We take this opportunity to correct a slight error in [YZ99]. In [YZ99, Theorem 6.2(1)] the complex $R$ should be defined as $R:=\left(\widetilde{R}_{t}\right)_{0}$, namely the degree 0 component of the localization with respect to the element $t$. The rest of that theorem (including the proof) is correct.

If $A$ is a differential $\mathbb{k}$-algebra of finite type, then so is the enveloping algebra $A^{\mathrm{e}}$. Hence, the rigid dualizing complex $R_{A^{\mathrm{e}}}$ exists, as does the rigid perverse t-structure on $\mathrm{D}_{\mathrm{f}}^{\mathrm{b}}\left(\operatorname{Mod} A^{\mathrm{e}}\right)$, whose heart is the category ${ }^{p} \mathrm{D}_{\mathrm{f}}^{\mathrm{b}}\left(\operatorname{Mod} A^{\mathrm{e}}\right)^{0}$ of perverse $A^{\mathrm{e}}$-modules.

TheOREm 8.9. Let $A$ be a differential $\mathbb{k}$-algebra of finite type with rigid dualizing complex $R_{A}$. Then $R_{A} \in{ }^{p} D_{\mathrm{f}}^{\mathrm{b}}\left(\operatorname{Mod} A^{\mathrm{e}}\right)^{0}$.

Proof. Consider the $\mathbb{k}$-algebra isomorphism

$$
\tau:\left(A^{\mathrm{op}}\right)^{\mathrm{e}}=A^{\mathrm{op}} \otimes A \stackrel{\simeq}{\longrightarrow} A \otimes A^{\mathrm{op}}=A^{\mathrm{e}}
$$

with formula $\tau\left(a_{1} \otimes a_{2}\right):=a_{2} \otimes a_{1}$. Given an $A^{\mathrm{e}}$-module $M$ let ${ }^{\tau} M$ be the $\left(A^{\mathrm{op}}\right)^{\mathrm{e}}$-module with action via $\tau$, i.e.

$$
\left(a_{1} \otimes a_{2}\right) \cdot_{\tau} m:=\tau\left(a_{1} \otimes a_{2}\right) \cdot m=a_{2} m a_{1}
$$

for $m \in M$ and $a_{1} \otimes a_{2} \in\left(A^{\mathrm{op}}\right)^{\mathrm{e}}$. Performing this operation on the complex $R_{A} \in \mathrm{D}\left(\operatorname{Mod} A^{\mathrm{e}}\right)$ we obtain a complex ${ }^{\tau} R_{A} \in \mathrm{D}\left(\operatorname{Mod}\left(A^{\mathrm{op}}\right)^{\mathrm{e}}\right)$. Each of the conditions in Definitions 4.1 and 4.10 is automatically verified, and hence $R_{A^{\mathrm{op}}}:={ }^{\tau} R_{A}$ is a rigid dualizing complex over $A^{\mathrm{op}}$.

According to Theorem 8.5 we get an isomorphism

$$
R_{A^{\mathrm{e}}} \cong R_{A} \otimes R_{A^{\mathrm{op}}}=R_{A} \otimes\left({ }^{\tau} R_{A}\right)
$$

in $\mathrm{D}\left(\operatorname{Mod}\left(A^{\mathrm{e}}\right)^{\mathrm{e}}\right)$. However, the left (respectively right) $A^{\mathrm{e}}$ action on $R_{A} \otimes\left({ }^{\tau} R_{A}\right)$ is exactly the outside (respectively inside) action on $R_{A} \otimes R_{A}$. By rigidity (cf. Definition 4.10) we have

$$
R_{A} \cong \operatorname{RHom}_{A^{\mathrm{e}}}\left(A, R_{A} \otimes R_{A}\right) \cong \operatorname{RHom}_{A^{\mathrm{e}}}\left(A, R_{A^{\mathrm{e}}}\right)
$$

in $\mathrm{D}\left(\operatorname{Mod} A^{\mathrm{e}}\right)$.

Finally, since $A^{\mathrm{e}} \cong\left(A^{\mathrm{e}}\right)^{\mathrm{op}}$, via the involution $\tau$, we may view $\operatorname{RHom}_{A^{\mathrm{e}}}\left(-, R_{A^{\mathrm{e}}}\right)$ as an autoduality of $\mathrm{D}_{\mathrm{f}}^{\mathrm{b}}\left(\operatorname{Mod} A^{\mathrm{e}}\right)$. By definition of the rigid t-structure this duality exchanges $\operatorname{Mod}_{\mathrm{f}} A^{\mathrm{e}}$ and ${ }^{p} \mathrm{D}_{\mathrm{f}}^{\mathrm{b}}\left(\operatorname{Mod} A^{\mathrm{e}}\right)^{0}$. Since $A \in \operatorname{Mod}_{\mathrm{f}} A^{\mathrm{e}}$ it follows that $R_{A} \in{ }^{p} \mathrm{D}_{\mathrm{f}}^{\mathrm{b}}\left(\operatorname{Mod} A^{\mathrm{e}}\right)^{0}$.

We know that the cohomology bimodules $\mathrm{H}^{i} R_{A}$ are central $\mathrm{Z}(A)$-modules. The next lemma is used a few times. 


\section{DifFERENTIAL ALGEBRAS}

Lemma 8.10. Let $A$ be a differential $\mathbb{k}$-algebra of finite type and $a \in A$ a noninvertible central regular element. Define $B:=A /(a)$. Let $R_{A}$ and $R_{B}$ denote the rigid dualizing complexes of $A$ and $B$, respectively. Then there is a long exact sequence

$$
\cdots \rightarrow \mathrm{H}^{i} R_{B} \rightarrow \mathrm{H}^{i} R_{A} \stackrel{a}{\rightarrow} \mathrm{H}^{i} R_{A} \rightarrow \mathrm{H}^{i+1} R_{B} \rightarrow \cdots
$$

of A-bimodules.

Proof. Trivially $A \rightarrow B$ is a finite centralizing homomorphism. By Proposition 8.2 the trace morphism $\operatorname{Tr}_{B / A}: R_{B} \rightarrow R_{A}$ exists. In particular, $R_{B} \cong \operatorname{RHom}_{A}\left(B, R_{A}\right)$. There is an exact sequence of bimodules

$$
0 \rightarrow A \stackrel{a}{\rightarrow} A \rightarrow B \rightarrow 0
$$

Applying the functor $\operatorname{RHom}_{A}\left(-, R_{A}\right)$ to it and taking cohomologies we obtain the long exact sequence we want.

Below we give a couple of examples of differential $\mathbb{k}$-algebras of finite type and their rigid dualizing complexes.

Example 8.11. Let $C$ be a smooth $n$-dimensional $\mathbb{k}$-algebra in characteristic 0 and $A:=\mathcal{D}(C)$ the ring of differential operators. Then the rigid dualizing complex is $R_{A}=A[2 n]$; see [Ye00].

Example 8.12. Let $\mathfrak{g}$ be an $n$-dimensional Lie algebra over $\mathbb{k}$ and $A:=\mathrm{U}(\mathfrak{g})$ its universal enveloping algebra. By [Ye00] the rigid dualizing complex is $R_{A}=A \otimes\left(\bigwedge^{n} \mathfrak{g}\right)[n]$, where $\bigwedge^{n} \mathfrak{g}$ has the adjoint $A$ action on the left and the trivial action on the right.

Suppose that $A$ is a ring with nonnegative exhaustive filtration $F$ such that the Rees ring $\widetilde{A}:=\operatorname{Rees}^{F} A$ is left noetherian. We recall that a filtered $(A, F)$-module $(M, F)$ is called good if it is bounded below, exhaustive and $\operatorname{Rees}^{F} M$ is a finite $\widetilde{A}$-module.

In the two previous examples the cohomology bimodules $\mathrm{H}^{i} R_{A}$ all came equipped with filtrations that were both differential and good on both sides. These properties turn out to hold in general, as Theorems 8.13 and 8.14 show.

Theorem 8.13. Let $A$ be a differential $\mathbb{k}$-algebra of finite type, and let $R_{A}$ be the rigid dualizing complex of $A$. Let $F$ be some differential $\mathbb{k}$-filtration of finite type of $A$. Then for every $i$ there is an induced filtration $F$ of $\mathrm{H}^{i} R_{A}$, such that $\left(\mathrm{H}^{i} R_{A}, F\right)$ is a good filtered $(A, F)$-module on both sides.

Proof. Define $\widetilde{A}:=\operatorname{Rees}^{F} A \subset A[t]$. Let $\widetilde{F}=\left\{\widetilde{F}_{i} \widetilde{A}\right\}$ be the filtration from Lemma 1.4. Then

$$
\operatorname{gr}^{\widetilde{F}} \widetilde{A} \cong\left(\operatorname{gr}^{F} A\right) \otimes \mathbb{k}[t]
$$

as $\mathbb{k}$-algebras. The center is

$$
\mathrm{Z}\left(\operatorname{gr}^{\widetilde{F}} \widetilde{A}\right) \cong \mathrm{Z}\left(\operatorname{gr}^{F} A\right) \otimes \mathbb{k}[t],
$$

which is a finitely generated commutative $\mathbb{k}$-algebra. Also $\operatorname{gr}^{\widetilde{F}} \widetilde{A}$ is a finite $\mathrm{Z}(\operatorname{gr} \widetilde{F} \widetilde{A})$-module. We conclude that $\widetilde{F}$ is a differential $\mathbb{k}$-filtration of finite type on $\widetilde{A}$. Moreover, each $\mathbb{k}$-submodule $\widetilde{F}_{i} \widetilde{A}$ is graded, where $\widetilde{A} \subset A[t]$ has the grading $F$ in which $\operatorname{deg}^{F}(t)=1$.

Applying Theorem 3.1 and Proposition 3.5 to the filtered $\mathbb{k}$-algebra $(\widetilde{A}, \widetilde{F})$, we obtain another filtration $\widetilde{G}$ on $\widetilde{A}$. This new filtration is also differential $\mathbb{k}$-filtration of finite type, and each $\mathbb{k}$-submodule $\widetilde{G}_{i} \widetilde{A} \subset \widetilde{A}$ is graded (for the grading $F$ ). Furthermore, gr $\widetilde{G} \widetilde{A}$ is a connected graded $\mathbb{k}$-algebra (when considered as $\mathbb{Z}$-graded ring with the grading $\widetilde{G}$ ).

Define

$$
B:=\operatorname{Rees}^{\widetilde{G}} \widetilde{A} \subset \widetilde{A}[s] \subset A[s, t] .
$$

This is a $\mathbb{Z}^{2}$-graded ring with grading $(\widetilde{G}, F)$, in which $\operatorname{deg}(\widetilde{G}, F)(s)=(1,0)$ and $\operatorname{deg}^{(\widetilde{G}, F)}(t)=(0,1)$. 


\section{A. YeKutieli And J. J. Zhang}

Consider the $\mathbb{k}$-algebra $B$ with its grading $\widetilde{G}$. This is a connected graded $\mathbb{k}$-algebra. The quotient $B /(s) \cong \operatorname{gr} \widetilde{G} \widetilde{A}$ is a finitely generated commutative $\mathbb{k}$-algebra. Therefore, by [YZ99, Theorem 5.13], $B$ has a balanced dualizing complex $R_{B} \in \mathrm{D}^{\mathrm{b}}\left(\operatorname{GrMod} B^{\mathrm{e}}\right)$. By [vdB97, Theorem 6.3] we get

$$
R_{B} \cong\left(\mathrm{R} \Gamma_{\mathfrak{m}} B\right)^{*}
$$

in $\mathrm{D}\left(\operatorname{GrMod} B^{\mathrm{e}}\right)$. Here $\Gamma_{\mathfrak{m}}$ is the torsion functor with respect to the augmentation ideal $\mathfrak{m}$ of $B$, and

$$
(M)^{*}:=\operatorname{Hom}_{\mathbb{k}}^{\mathrm{gr}}(M, \mathbb{k})=\bigoplus_{i} \operatorname{Hom}_{\mathbb{k}}\left(M_{-i}, \mathbb{k}\right),
$$

the graded dual of the graded $\mathbb{k}$-module $M$. In particular, for every $p$ there is an isomorphism of $B$-bimodules $\mathrm{H}^{-p} R_{B} \cong\left(\mathrm{H}_{\mathfrak{m}}^{p} B\right)^{*}$ where

$$
\mathrm{H}_{\mathfrak{m}}^{p} B:=\mathrm{H}^{p} \mathrm{R} \Gamma_{\mathfrak{m}} B \cong \lim _{k \rightarrow} \operatorname{Ext}_{B}^{p}\left(B / \mathfrak{m}^{k}, B\right)
$$

Now for each $k$

$$
\operatorname{Ext}_{B}^{p}\left(B / \mathfrak{m}^{k}, B\right)=\bigoplus_{(i, j) \in \mathbb{Z}^{2}} \operatorname{Ext}_{B}^{p}\left(B / \mathfrak{m}^{k}, B\right)_{(i, j)}
$$

where $(i, j)$ is the $(\widetilde{G}, F)$ degree. Therefore in the direct limit we get a double grading

$$
\mathrm{H}_{\mathfrak{m}}^{p} B=\bigoplus_{(i, j) \in \mathbb{Z}^{2}}\left(\mathrm{H}_{\mathfrak{m}}^{p} B\right)_{(i, j)} .
$$

Since for every $i$ the $\mathbb{k}$-module $\left(\mathrm{H}_{\mathfrak{m}}^{p} B\right)_{i}=\bigoplus_{j \in \mathbb{Z}}\left(\mathrm{H}_{\mathfrak{m}}^{p} B\right)_{(i, j)}$ is finite it follows that the graded dual $\left(\mathrm{H}_{\mathfrak{m}}^{p} B\right)^{*}$, which is computed with respect to the $\widetilde{G}$ grading, is also $\mathbb{Z}^{2}$-graded. We see that $\mathrm{H}^{-p} R_{B}$ is in fact a $\mathbb{Z}^{2}$-graded $B$-bimodule.

By [YZ99, Theorem 6.2] the complex $R_{\widetilde{A}}:=\left(\left(R_{B}[-1]\right)_{s}\right)_{0}$ is a rigid dualizing complex over the $\operatorname{ring} \widetilde{A} \cong B /(s-1)$; cf. Remark 8.8. Hence, each cohomology

$$
\mathrm{H}^{p} R_{\widetilde{A}} \cong \frac{\mathrm{H}^{p-1} R_{B}}{(s-1) \cdot \mathrm{H}^{p-1} R_{B}}=\operatorname{sp}_{1}^{\widetilde{G}} \mathrm{H}^{p-1} R_{B}
$$

is a graded $\widetilde{A}$-bimodule (with the $\mathbb{Z}$-grading $F$ in which $\operatorname{deg}^{F}(t)=1$ ), finite on both sides.

Next we have $A \cong \widetilde{A} /(t-1)$. Because $t-1$ is a central regular non-invertible element, Lemma 8.10 states there is an exact sequence

$$
\cdots \rightarrow \mathrm{H}^{p} R_{A} \rightarrow \mathrm{H}^{p} R_{\widetilde{A}} \stackrel{t-1}{\longrightarrow} \mathrm{H}^{p} R_{\widetilde{A}} \rightarrow \mathrm{H}^{p+1} R_{A} \rightarrow \mathrm{H}^{p+1} R_{\widetilde{A}} \rightarrow \cdots
$$

of $A$-bimodules. Since $\mathrm{H}^{p} R_{\widetilde{A}}$ is graded, the element $t-1$ is a nonzero divisor on it, and therefore we get an exact sequence

$$
0 \rightarrow \mathrm{H}^{p-1} R_{\widetilde{A}} \stackrel{t-1}{\longrightarrow} \mathrm{H}^{p-1} R_{\widetilde{A}} \rightarrow \mathrm{H}^{p} R_{A} \rightarrow 0
$$

Thus, the bimodule

$$
\mathrm{H}^{p} R_{A} \cong \frac{\mathrm{H}^{p-1} R_{\widetilde{A}}}{(t-1) \cdot \mathrm{H}^{p-1} R_{\widetilde{A}}}=\operatorname{sp}_{1}^{F} \mathrm{H}^{p-1} R_{\widetilde{A}}
$$

inherits a bounded below exhaustive filtration $F$, and $\operatorname{Rees}\left(\mathrm{H}^{p} R_{A}, F\right)$, being a quotient of $\mathrm{H}^{p-1} R_{\widetilde{A}}$, is a finite $\widetilde{A}$-module on both sides. By definition $\left(\mathrm{H}^{p} R_{A}, F\right)$ is then is a good filtered $(A, F)$-module on both sides.

Theorem 8.14. Let $C$ be a finitely generated commutative $\mathbb{k}$-algebra, let $A$ be differential $C$-ring of finite type, and let $R_{A}$ be the rigid dualizing complex of $A$. Then for every $i$ the $C$-bimodule $\mathrm{H}^{i} R_{A}$ is differential. 


\section{DifFERENTIAL ALGEBRAS}

Proof. Using the same setup as in the proof of Theorem 8.13, define

$$
\bar{A}:=\operatorname{gr}^{F} A=\operatorname{sp}_{0}^{F} \widetilde{A}=\widetilde{A} /(t) .
$$

So $\bar{A}$ is a $C$-algebra. Let $R_{\bar{A}}$ be the rigid dualizing complex of $\bar{A}$. By [YZ99, Corollary 3.6] the $\bar{A}$-bimodule $\mathrm{H}^{i} R_{\bar{A}}$ is $Z(\bar{A})$-central, and hence it is a central $C$-bimodule. According to Lemma 8.10 there is an exact sequence of $\widetilde{A}$-bimodules

$$
\mathrm{H}^{i-1} R_{\widetilde{A}} \stackrel{t}{\rightarrow} \mathrm{H}^{i-1} R_{\widetilde{A}} \rightarrow \mathrm{H}^{i} R_{\bar{A}} .
$$

Therefore,

$$
\mathrm{sp}_{0}\left(\mathrm{H}^{i-1} R_{\widetilde{A}}\right)=\left(\mathrm{H}^{i-1} R_{\widetilde{A}}\right) / t \cdot\left(\mathrm{H}^{i-1} R_{\widetilde{A}}\right) \hookrightarrow \mathrm{H}^{i} R_{\bar{A}}
$$

is a central $C$-bimodule.

To conclude the proof, consider the filtration $F$ of $\mathrm{H}^{i} R_{A}$ from Theorem 8.13. Because $\left(\mathrm{H}^{i} R_{A}, F\right) \cong \operatorname{sp}_{1}\left(\mathrm{H}^{i-1} R_{\widetilde{A}}\right)$ is a good filtered $(A, F)$-module, say on the left, we see that $\left(\mathrm{H}^{i} R_{A}, F\right)$ is exhaustive and bounded below. Now

$$
\operatorname{Rees}\left(\mathrm{H}^{i} R_{A}, F\right) \cong\left(\mathrm{H}^{i-1} R_{\widetilde{A}}\right) /\{t \text {-torsion }\},
$$

so $\operatorname{gr}\left(\mathrm{H}^{i} R_{A}, F\right)$ is a quotient of $\operatorname{sp}_{0}\left(\mathrm{H}^{i-1} R_{\widetilde{A}}\right)$. It follows that $\operatorname{gr}\left(\mathrm{H}^{i} R_{A}, F\right)$ is a central $C$-bimodule. Thus, $F$ is a differential $C$-filtration of $\mathrm{H}^{i} R_{A}$.

Corollary 8.15. In the situation of Theorem 8.14, let $U:=\operatorname{Spec} C$. Given an affine open set $V \subset U$ let $C^{\prime}:=\Gamma\left(V, \mathcal{O}_{U}\right)$. Then $A^{\prime}:=C^{\prime} \otimes_{C} A \otimes_{C} C^{\prime}$ is a noetherian $\mathbb{k}$-algebra, $A \rightarrow A^{\prime}$ is flat and $R^{\prime}:=A^{\prime} \otimes_{A} R_{A} \otimes_{A} A^{\prime}$ is an Auslander rigid dualizing complex over $A^{\prime}$.

Proof. According to Corollary 5.20, $A^{\prime}$ is a noetherian $\mathbb{k}$-algebra and $A \rightarrow A^{\prime}$ is a localization. We know that $A^{\mathrm{e}}$ is noetherian. By Theorem 8.14 each of the cohomology bimodules $\mathrm{H}^{i} R_{A}$ are differential as $C$-bimodules, hence by Proposition 5.17 they are evenly localizable to $C^{\prime}$. Thus, all of the hypotheses of Theorem 6.2(1)-(3) are satisfied.

\section{ACKNowledGements}

The authors wish to thank Joseph Bernstein, Sophie Chemla, Masaki Kashiwara, Thierry Levasseur, Zinovy Reichstein, Paul Smith and Michel van den Bergh for helpful conversations.

\section{REFERENCES}

ATV91 M. Artin, J. Tate and M. van den Bergh, Modules over regular algebras of dimension 3, Invent. Math. 106 (1991), 335-388.

Bj89 J. E. Björk, The Auslander condition on noetherian rings, in Séminaire Dubreil-Malliavin 1987-88, Lecture Notes in Mathematics, vol. 1404 (Springer, Berlin, 1989), 137-173.

BBD81 A. A. Beilinson, J. Bernstein and P. Deligne, Faisceaux pervers, in Analysis and topology on singular spaces, I (Luminy, 1981), Astérisque 100 (1982), 5-171.

Ch99 S. Chemla, A duality property for complex Lie algebroids, Math. Z. 232 (1999), 367-388.

Ch04 S. Chemla, Rigid dualizing complexes for quantum enveloping algebras and algebras of generalized differential operators, J. Algebra 276 (2004), 80-102.

EG67 A. Grothendieck and J. Dieudonné, Éléments de géometrie algébrique, IV. Publ. Math. Inst. Hautes Études Sci. 32 (1967).

EG71 A. Grothendieck and J. Dieudonné, Éléments de géometrie algébrique, I (Springer, Berlin, 1971).

Ha66 R. Hartshorne, Residues and duality, Lecture Notes in Mathematics, vol. 20 (Springer, Berlin, 1966).

Ha77 R. Hartshorne, Algebraic geometry (Springer, New York, 1977). 


\section{DifFERENTIAL ALGEBRAS}

Jo98 P. Jørgensen, Non-commutative graded homological identities, J. London Math. Soc. (2) 57 (1998), $336-350$.

KL00 G. R. Krause and T. H. Lenagan, Growth of algebras and Gelfand-Kirillov dimension, revised edition, Graduate Studies in Mathematics, vol. 22 (American Mathematical Society, Providence, RI, 2000).

Ka03 M. Kashiwara, $t$-structures on the derived categories of holonomic $\mathcal{D}$-modules and coherent $\mathcal{O}$-modules, Preprint (2003), math.AG/0302086.

KS90 M. Kashiwara and P. Schapira, Sheaves on manifolds (Springer, Berlin, 1990).

Le92 T. Levasseur, Some properties of noncommutative regular rings, Glasgow Math. J. 34 (1992), $277-300$.

MR87 J. C. McConnell and J. C. Robson, Noncommutative noetherian rings (Wiley, Chichester, 1987).

MS89 J. C. McConnell and J. T. Stafford, Gelfand-Kirillov dimension and associated graded modules, J. Algebra 125 (1989), 197-214.

MY01 J. Miyachi and A. Yekutieli, Derived Picard groups of finite dimensional hereditary algebras, Compositio Math. 129 (2001), 341-368.

Ri63 G. S. Rinehart, Differential forms on general commutative algebras, Trans. Amer. Math. Soc. 108 (1963), 195-222.

Si67 L. Silver, Noncommutative localizations and applications, J. Algebra 7 (1967), 44-76.

vdB97 M. van den Bergh, Existence theorems for dualizing complexes over non-commutative graded and filtered rings, J. Algebra 195 (1997), 662-679.

WZ00 Q. S. Wu and J. J. Zhang, Some homological invariants of local PI algebras, J. Algebra 225 (2000), 904-935.

Ye92 A. Yekutieli, Dualizing complexes over noncommutative graded algebras, J. Algebra 153 (1992), 41-84.

Ye96 A. Yekutieli, The residue complex of a noncommutative graded algebra, J. Algebra 186 (1996), $522-543$.

Ye99 A. Yekutieli, Dualizing complexes, Morita equivalence and the derived Picard group of a ring, J. London Math. Soc. (2) 60 (1999), 723-746.

Ye00 A. Yekutieli, The rigid dualizing complex of a universal enveloping algebra, J. Pure Appl. Algebra 150 (2000), 85-93.

YZ99 A. Yekutieli and J. J. Zhang, Rings with Auslander dualizing complexes, J. Algebra 213 (1999), $1-51$.

YZ02 A. Yekutieli and J. J. Zhang, Dualizing complexes and perverse sheaves on noncommutative ringed schemes, Preprint (2002), math.AG/0211309.

YZ03 A. Yekutieli and J. J. Zhang, Residue complexes over noncommutative rings, J. Algebra 259 (2003), 451-493.

Amnon Yekutieli amyekut@math.bgu.ac.il

Department of Mathematics, Ben Gurion University, Be'er Sheva 84105, Israel

James J. Zhang zhang@math.washington.edu

Department of Mathematics, Box 354350, University of Washington, Seattle, WA 98195, USA 